\title{
Proceedings of the II International Conference on Food Chemistry \& Technology (FCT-2016)
}

\section{Keynote Presentations}

\section{Optimizing the Design of Preservation Processes}

\author{
Dennis R. Heldman
}

The Obio State University, Columbus, OH, USA

\begin{abstract}
Since many traditional food preservation technologies have negative influence on food quality attributes, more careful analyses of the processes are required. Thermal processes influence several of the components of a food, and cause changes in physical structure as well as other sensory attributes. Often, these processes can be designed to ensure the desired outcomes in terms of preservation, and still minimize the influence on key quality attributes. The overall objective of this presentation is to discuss process models required to maximize quality retention, while meeting the goals of preservation. For these applications, an optimization model depends on two elements. The first is the kinetic models needed to describe the influence of the preservation process on shelf-life limiting components of the product, as well as the influence of the process on one or more quality attributes. The second element is the transport model needed to describe the distribution of preservation process intensities within the product. The integration of these models predicts the inactivation of the shelf-life limiting components and the retention of quality attributes during of the preservation process.
\end{abstract}

The optimum process is defined as the process with the desired outcome in terms of shelf life and/or safety, while achieving maximum retention of one or more product quality attributes. In order to identify the optimum process, the integrated models must be evaluated over a range processes providing the same shelf life or level of safety. The range of processes includes an array of higher process intensities and corresponding lower process times. An obvious situation for optimization occurs during thermal processes for a food in a cylindrical container, when the influence of the process on quality attributes varies significantly from regions near the surface of the container, as compared to interior regions of the product. This and similar process models will be discussed.

\section{Antibiotic Activities of Food-Compatible Compounds and Plant Extracts against Non- Resistant and Antibiotic-Resistant Foodborne Pathogens}

\section{Mendel Friedman}

Healthy Processed Foods Research, Western Regional Research Center, Agricultural Research Service, United States Department of Agriculture, Washington, DC, USA

\begin{abstract}
Foodborne pathogenic bacteria include strains of Bacilli, Campylobacter, Salmonella, and Staphylococci. The antibiotic resistance of pathogens is of particular concern. Bacteria can exert adverse effects in tissues in at least one of two ways: adhesion to and penetration into cells and release of cellular toxins. There has been interest in developing new types of effective and non-toxic antimicrobial compounds. Some promising natural antimicrobials include polyphenols from food plants such as tea, apples, grapes or pomegranates, chitosan from crustaceans, and essential oils from aromatic plants. The objective of this presentation is largely limited to summarizing our collaborative studies on the inhibition of growth of foodborne pathogens
\end{abstract}


in vitro in/on food, and in vivo. The ultimate goal of these studies is to discover practical food formulations that use the active compounds to reduce pathogens in foods, feeds, food animals and humans after consumption. In this presentation I will present brief overviews of the following relevant aspects: (a) susceptibilities of different the bacteria to inactivation; (b) inactivation of pathogens on contaminated leafy greens and meat; (c) concurrent inhibition of bacteria and carcinogenic heterocyclic amines in cooked hamburgers; (d) development of antimicrobial wine marinades; (e) efficacy of antimicrobial edible films prepared from fruits and vegetables on leafy greens and meat products that the food industry and consumers could use as wrappings to control surface contamination; and (f) protection of mice against salmonellosis through the stimulation of the immune system. Edible plant compounds are a useful source of antimicrobials that can protect against both nonresistant and resistant pathogens.

\title{
In vitro Models for Cost-Effective Screening of the Bioaccessibility and Bioavailability of Health Promoting Compounds from Foods and Ingredients
}

\author{
Mark Failla \\ The Obio State University, Columbus, OH, USA
}

\begin{abstract}
Although there are only 42 essential nutrients required for the development and reproduction of humans, it has been estimated that plant foods contain at least 20,000 distinct compounds. Many of the "non-essential" compounds and/or their metabolites generated by the gut microbiota and host tissues are known to possess health-promoting molecular and cellular activities. Efficacy of these dietary compounds is dependent on both the amount consumed and the efficiency with which the compound or its bioactive metabolites are delivered to target tissues, i.e., their bioavailability. Bioavailability requires release of the compound from the food matrix in order to be accessible for uptake by absorptive gut epithelial cells. Release of dietary compounds and particularly dietary lipophilic compounds from the food matrix during digestion is affected by numerous factors, including variety of the plant, pre-harvest conditions, post-harvest processing, styles of food preparation and other components in a meal, as well as host genotype and physiological status. While randomly controlled, double blind investigations with human subjects represent the gold standard for determination of the extent of absorption of ingested compounds, systematic investigation of effects of biotic and abiotic factors on accessibility to the gut epithelium and microbiota requires use of costeffective, relatively high throughput methods. In vitro models that simulate digestion of foods and ingredients and the Caco-2 human intestinal cell model for determining the bioaccessibility, metabolism and bioavailability of dietary compounds with health-promoting activities will be discussed. Several examples of the use of such methods will be presented.
\end{abstract}

\section{Changes of Soybean Biochemical Components and Food Quality as Affected by Post- Harvest Storage Conditions}

\author{
Sam K. C. Chang \\ Department of Food Science, Nutrition, and Health Promotion, Mississippi State University, Mississippi State, MS, USA
}

\begin{abstract}
Soy food quality is greatly affected by the chemical components in soybean materials. During storage of soybeans, the biochemical components change, and these changes affect the final food quality. In addition to microbiological changes, storage under various humidities and temperatures may induce undesirable changes in proteins, lipids, phytate, titratable acidity, water absorption and isoflavones, and lead to deterioration of food quality. In general, protein stored under undesirable conditions form large aggregates in the seeds through the formation of disulfide linkages and hydrophobic interactions, and leading to water-unextractable aggregates, and those remain extractable have changes in secondary structures that lead to weak tofu gel formation. Undesirable storage conditions also increased titratable acids and oxidized lipids, hydrolyzed phytate, and decreased water absorption, decreased sugar contents and converted conjugated isoflavones to aglycones. On the other hand, soybean stored under cold climate, and low humidity can keep the quality longer than 18 months for making soymilk and tofu. This presentation will review primarily the work on how storage affects protein chemistry and the food quality associated with protein changes.
\end{abstract}




\title{
A New Era for Prebiotics: Improving Activation of Bioactive Food Components by the Microbiome
}

\author{
Elizabeth Jeffery \\ University of Illinois, Champaign, IL, USA
}

\begin{abstract}
Prebiotics are frequently defined as nondigestible food ingredient that promote the growth of beneficial microorganisms in the gut. Research over the last few years has turned this on its head, giving a broader definition that prebiotics are food ingredients that reach the colon where they interact with the microbiome, frequently altering the abundance and/or activity of specific microorganisms and producing absorbable metabolites with health benefits. This talk will discuss the role of the microbiome in formation of bioactive metabolites from fruits and vegetables, extending past short chain fatty acids to flavonoids and isothiocyanates.

Isothiocyanates protect brassica vegetables against insect herbivores, being released from their parent glucosinolates by the plant enzyme myrosinase when herbivores chew the plant. These same isothiocyanates can trigger a defense response in mammals, including humans - leading to protection against cancer. However, most people like to cook their broccoli and other brassica vegetables, which destroys myrosinase. Although human tissue has no suitable hydrolyzing enzyme - a thiohydrolase - recent studies show that the gut microbiota can perform this action - and do so far better when brassica vegetables are introduced into the daily diet. The need for future studies evaluate the role of the microbiome in optimizing the health benefits that we gain from fruits and vegetables will be discussed.
\end{abstract}

\section{A Novel Highly-Sensitive Inorganic Arsenic Speciation in Rice by a Gas Chromatography Coupled to Tandem Mass Spectrometry in Combination with One Step Derivatization Technique}

\author{
Mun YhungJung \\ Department of Food Science and Biotechnology, Graduate School, Woosuk University, Wanju, Jeonbuk, Republic of Korea
}

\begin{abstract}
Arsenic is a highly toxic metalloid ubiquitously present in the environment such as soil and waters. The arsenic in water and soil is transferred to plant crops and enters the food chain. Rice has been recognized as a significant dietary source of arsenics. We developed a new fast and selective analytical method for the determination of inorganic arsenic (iAs) in rice by a gas chromatography - tandem mass spectrometry (GC-MS/MS) in combination with one step derivatization of iAs with British Anti-Lewsite (BAL). Two step derivatization of iAs with BAL has been previously performed for the GC-MS analysis. In this paper, the quantitative one step derivatization condition was successfully established. A fast and sensitive GC-MS/MS was established with a short nonpolar capillary column under the fast oven temperature ramp rate $\left(4^{\circ} \mathrm{C} / \mathrm{s}\right)$ and high linear velocity $(108.8 \mathrm{~cm} / \mathrm{sec})$ of the carrier gas. The established GC-MS/MS method showed an excellent linearity in a wide range, ultralow limit of detection (LOD, $0.08 \mathrm{pg}$ ), and high precision and accuracy. The GC-MS/MS technique showed 22.5-fold higher sensitivity on iAs analysis than GC-MS method. The gas chromatographic retention of iAs was 1.98 min. The established method was successfully applied to quantify the iAs contents in Korean polished rice. The mean iAs content in the Korean polished rice $(\mathrm{n}=27)$ was $66.1 \mu \mathrm{g} \mathrm{kg}^{-1}$ with the range of $37.5-125.0 \mu \mathrm{g} \mathrm{kg}{ }^{-1}$. This represents the first report on the GC-tandem mass spectrometry in combination with the one step derivatization with BAL for the iAs speciation in rice. This GC-MS/MS method would be a simple, reliable and valuable tool for the iAs analysis in rice in the laboratories in which the expensive and element specific HPLC-ICP-MS is not available.
\end{abstract}




\title{
Glass-Transition Boundary Curve for Shelf-Life Prediction
}

\author{
Martin Buehler, Zach Campbell and Brady Carter \\ Decagon Devices Inc., 2365 NE Hopkins Ct, Pullman, WA, USA
}

\begin{abstract}
The Glass-Transition Boundary Curve (GTBC) was used to identify regions in the Tg versus aw plane where compounds have a short and long shelf life. This method is applicable to compounds that undergo glass transition where compounds change from the glassy to rubbery state. These compounds are characterized by a drift in electrical and/or thermal properties when exposed to elevated temperatures and relative humidity. The methodology presented here is based on the drift of properties measured using dielectric relaxation spectroscopy (DRS) [1]. A simple algorithm has been developed for extracting the Boundary Curve from DRS measurements on sprayed-dried powders that are initially in a stressed state and relax given proper thermal and relative humidity conditions. The use of the GTBC method for predicting shelf life will be illustrated using skim milk powder where the amount of protein is clearly visible in samples that have from 30 to $90 \%$ protein. In addition, the presence of melamine has been detected using DRS where $1 \mathrm{ppm}$ melamine has been measured in wet-blended skim milk powder. The apparatus for obtaining the measurements will also be described.
\end{abstract}

\section{Reference}

1. Buehler MG, Kindle ML, Carter BP. 2015. Using dielectric relaxation spectroscopy to characterize the glass transition time of polydextrose. J Food Sci 80(6): 1243-1252. doi: 10.1111/1750-3841.12887

\section{Reduction of Cancer Risk Related to the Consumption of Red Meat}

\author{
Isabel M.P.L.V.O. Ferreira ${ }^{1 *}$, Olga Viegas ${ }^{1,2}$, Armindo Melo ${ }^{1}$, Miguel Faria ${ }^{1}$ and Olívia Pinho ${ }^{1,2}$ \\ ${ }^{1}$ LAQV/REQUIMTE, Laboratório de Bromatologia e Hidrologia, Departamento de Ciências Químicas, Faculdade de Farmácia, \\ Universidade do Porto, Portugal \\ ${ }^{2}$ Faculdade de Ciências da Nutrição e Alimentação, Universidade do Porto, Portugal
}

\begin{abstract}
The effects of red meat on health have been intensively studied by the scientific community. In October 2015, the International Agency for Research on Cancer released a report about carcinogenicity of consumption of red and processed meat. Increased cancer risk is mainly attributed to harmful compounds that are formed when the meat is processed, due to the formation of heat generated carcinogenic compounds, namely heterocyclic aromatic amines (HAs) and polycyclic aromatic hydrocarbons (PAHs). The goals of this work were (i) estimation of intake of HAs and PAHs from cooked meat (pan-fried and charcoal grilled beef, loin pork and chicken) by its quantification in meat samples cooked at home and taken from restaurants; (ii) mitigate the formation of those carcinogenic compounds by the use of wine and beer marinades; (iii) investigate the stability of most relevant heat generated carcinogenic compounds under gastro-intestinal conditions and their release from food matrix by simulation of an in vitro digestion. Variable amounts of HAs were quantified in pan fried, grilled and barbecued red meat, poultry and fish. PAHs were detected and quantified only in barbecued samples. More than $2 \mu \mathrm{g} / 100 \mathrm{~g}$ of HAs and PAHs can be taken in a single meal of barbecued meat. Mitigation of HAs and PAHs formation was achieved marinating meat with dark beer before grilling, a reduction of $90 \%$ of HAs and $53 \%$ of PAHs was observed. After in vitro digestion, the most abundant $\mathrm{HA}(\mathrm{PhIP})$ was less bioaccessible (only 7\%) than the PAH representative bezo[a]pyrene (BaP) ( 30\%).
\end{abstract}




\title{
Featured Presentations
}

\section{The Biological Activities and Phenolic Profiles of Salvia steminea and Salvia krononburgei from Anatolia}

\author{
Hava Batıbay ${ }^{1}$, Abdulselam Ertaş$^{2}$, Elif Varhan Oral ${ }^{3}$, Mehmet Boğa ${ }^{4}$, Mustafa Abdullah Yılmaz ${ }^{3}$, Isil Gazioglu ${ }^{5,6^{*}}$, Hamdi \\ Temel $^{3}$, Berrin Ziyadanoğullari ${ }^{1}$ and Mehmet Firat ${ }^{7}$ \\ ${ }^{1}$ Dicle University, Faculty of Science, Department of Analytical Chemistry, Turkey \\ ${ }^{2}$ Dicle University, Faculty of Pharmacy, Department of Pharmacognosy, Turkey \\ ${ }^{3}$ Dicle University, Faculty of Pharmacy, Department of Pharmaceutical and Analytical Chemistry, Turkey \\ ${ }^{4}$ Dicle University, Faculty of Pharmacy, Department of Pharmaceutical Technology, Turkey \\ ${ }^{5} U$. S. Food and Drug Administration, National Center for Toxicological Research, Jefferson, AR, USA \\ ${ }^{6}$ Bezmialem Vakif University, Faculty of Pharmacy, Department of Analytical Chemistry, Turkey \\ ${ }^{7} Y \ddot{z} z$ üncü Yıl University, Faculty of Education, Department of Biology, Turkey
}

\begin{abstract}
Salvia L. (Lamiaceae) genus is represented more than 900 species on the world and about 98 species in Turkey. Fifty-three of them are endemic. Salvia genus is a member of the Lamiaceae family. These plants have trichomes and aromatic qualities, due to containing of various essential oils used in perfumery and medicine. Medicinal plants are biologically active materials traditionally used in the treatment of a variety of diseases since ancient times. Essential oils derived from many plant species are known to have antimicrobial, antioxidant and anticholinesterase activities, and attempts to characterize their bioactive principles have increased in many pharmaceutical and food-processing applications. The aim of this study was to determine the chemical profile of different parts of (root, leaf, flower, branch) Salvia steminea and Salvia krononburgei by LC-MS/MS. Additionally, the total phenolic and flavonoid contents, antioxidant (DPPH free radical scavenging activity, beta carotene bleaching assay, CUPRAC, ABTS + .cation radical scavenging activity) and anticholinesterase (acetyl- and butrylkolinesterase enzymes) activities of these Salvia species were tested. The ethanol extract of root of $S$. steminea showed high antioxidant and anticholinestrase activities in all assays. In particular, it was determined that the high antioxidant potential of $S$. steminea showed a good correlation with high rosmarinic and caffeic acid content. Among the thirty-seven compounds studied by LC-MS/MS, fumaric acid and malic acid was found to be the most abundant compounds in all parts of Salvia steminea, Salvia krononburgei respectively. Besides, HPLC-FLD was used to determine total aflatoxin in the plant materials and any contamination was not found in the plants.
\end{abstract}

Acknowledgements: The research was funded by grant: DUBAP 14-FF-72 from Dicle University Scientific Research Projects Coordination Unit.

\section{Physicochemical Stability of Whey Protein Stabilized Fish Oil-in-Water Emulsions Containing Xanthan/Enzymatically Modified Guar Polysaccharide Mixtures}

\author{
John Khouryieh \\ Western Kentucky University, 1906 College Heights Blvd, Bowling Green, KY, USA
}

\begin{abstract}
Oil-in-water emulsions have been widely used as delivery systems of polyunsaturated fatty acids into foods. However, the thermodynamic instability of oil and water, and high susceptibility of unsaturated fatty acids to oxidation lead to physical and oxidative stability in oil-in-water emulsions. The objective of this study was to investigate the effect of xanthan/enzymatically modified guar polysaccharide mixtures on the physical and oxidative stability of whey protein isolate stabilized oil-in-water emulsions containing 20\% v/v menhaden fish oil. Emulsions were prepared using guar gum (GG), xanthan gum (XG), xanthan/ guar (XG/GG), and xanthan/enzyme-modified guar (XG/EMG) gum mixtures at $0,0.05,0.1,0.15,0.2$, and $0.3 \%$ gum concentrations. Enzymatic modified guar gum was obtained by hydrolyzing native guar gum using $\alpha$-galactosidase enzyme. Emulsions were evaluated for creaming, viscosity, particle size, and microstructure to evaluate the physical stability, and peroxide value, TBARS value and GC-MS solid phase micro extraction (SPME) experiments were performed to evaluate the oxidative stability. Emulsions containing XG/EMG gum mixtures exhibited better creaming stability and higher viscosity of all the emulsion types. However, increasing concentrations (0-0.3 wt \%) of XG/EMG mixtures did not affect the droplet size of the emulsions. The microstructures revealed decreased flocculation at higher concentrations. The primary and secondary lipid oxidation measurements indicated that emulsions containing XG/EMG mixtures were more effective in retarding the lipid
\end{abstract}


oxidation compared to XG, Guar (GG), and XG/GG gum mixtures. These results suggest that XG/EMG gum mixtures can be used as emulsifiers in oil-in-water emulsions to enhance both physical and oxidative stability of foods.

\title{
Determination of the Authenticity of Food Products by Infrared and Fluorescence Spectroscopies Coupled with Chemometric Tools
}

\author{
Romdhane Karoui \\ Artois University, Charles Viollette Institute, France
}

\begin{abstract}
Today, the International market is saturated with food products. The new challenge is not to produce a Standard product, which is not only differentiated by price, but also have unique characteristic and meet consumers' expectation. Two approaches can be used to reach these goals. On the one hand, fulfilling consumer demands can be met through the creation of commercial brand products with unique texture, flavour or usage appeal characteristics. On the other hand, protected designation of origin (PDO) and/or Protected Geographical Indication (PGI) make such foods very specific and well differentiated from others. Food adulteration has been practiced since a long time ago and becomes increasingly in the last years more sophisticated. Foods and ingredients presenting high-value are the most vulnerable for adulteration. Determination of food authenticity and detection of adulteration have become an important question in quality control and safety of food products. Although the physicochemical analyses are promising techniques to determine the authenticity of food products, they are time consuming and use several pollutant reagents. For these reasons, there is a need to develop rapid, inexpensive and efficient analytical methods for the detection of frauds and authentication of food products. Recently, more attention has been paid to the development of noninvasive and non-destructive techniques such as infrared, fluorescence, and so on. These techniques are fast, of relatively low cost, environmentally friendly, and provide a great deal of information with only one test, making them suitable for on-line and/or atline process control. In addition, the above-mentioned techniques often require little or no sample preparation and are relatively easy to operate. The potential and ease of application of these rapid techniques coupled with chemometric tools that have found new applications in the field of authentication will be covered. The use of specific techniques (front-face fluorescence, near and mid infrared) coupled with chemometric tools for the determination of the authenticity and integrity of food (e.g., milk, cheese, fish, egg, honey, etc.) will also be illustrated.
\end{abstract}

\section{The Unknown Nutrient: Discovery of the Health Benefits of Dietary Nitrate}

\author{
Nathan S. Bryan \\ Baylor College of Medicine, 1 Baylor Plaza, Houston, TX, USA
}

\begin{abstract}
Advancements in nutrition and discoveries of new nutrients have had revolutionary effects on health and disease management throughout history. The word nutrition itself means the process of nourishing or being nourished, especially the process by which a living organism assimilates food and uses it for growth and replacement of tissues. A nutrient is defined as any substance that nourishes an organism and to nourish is to sustain with food or nutriment; supply with what is necessary for life, health, and growth. Nutrients, therefore, are substances that are essential to life which must be supplied by food. We and others have recently discovered a new set of nutrients, lack of which may be the cause of all chronic diseases, including cardiovascular disease, the number one killer of men and women worldwide [1,2]. To the contrary, sufficient ingestion or supplementation of which may prevent many of these chronic diseases. Dietary nitrate, found primarily in green leafy vegetables, has been found to have enormous health benefits and is the likely mechanism of action for the Mediterranean Diet, The Dietary Approaches to Stop Hypertension (DASH) and the French Paradox [1, 3, 4]. We will review the current state of the science surrounding nitrate rich foods and present the published data on the therapeutic effects of dietary nitrate. We will also present foods and diets enriched in nitrate that physician can begin to recommend to their patients as a first line defense for nitric oxide insufficiency and the resulting cardiovascular issues. The audience will learn the challenges and opportunities that exist in understanding the variability in nutrient density of certain foods and how to make better recommendations to their patients.
\end{abstract}

\section{References}

1. Hord NG, Tang Y, Bryan NS. 2009. Food sources of nitrates and nitrites: the physiologic context for potential health benefits. Am J Clin Nutr 90(1): 1-10. doi: 10.3945/ajcn.2008.27131 
2. Bryan NS, Ivy JL. 2015. Inorganic nitrite and nitrate: evidence to support consideration as dietary nutrients. Nutr Res 35(8): 643-654. doi: 10.1016/j.nutres.2015.06.001

3. Lundberg JO, Feelisch M, Björne H, Jansson EA, Weitzberg E. 2006. Cardioprotective effects of vegetables: is nitrate the answer? Nitric Oxide 15(4): 359-362. doi: 10.1016/j.niox.2006.01.013

4. Lundberg JO, Gladwin MT, Ahluwalia A, Benjamin N, Bryan NS, et al. 2009. Nitrate and nitrite in biology, nutrition and therapeutics. Nat Chem Biol 5(12): 865-869. doi: 10.1038/nchembio.260

\title{
Plasmalogens - Neglected Nutrients with Crucial Biological Role
}

\author{
Varuzhan Sarkisyan*, Irina Glazkova, Alla Kochetkova, Yuliya Sidorova and Vera Kdentsova \\ Federal Research Centre of Nutrition and Biotechnology, Russia
}

\begin{abstract}
Plasmalogens are glycerophospholipids with a distinctive feature that is the presence of an ether linkage with a conjugated double bond in the sn-1 position. The structure, chemical properties, and data on the distribution of their in various tissues, have been studied since the 50 s of the $20^{\text {th }}$ century, however, only in the recent decades in-depth study on their biological role has become possible. The biological roles of plasmalogens as it is understood are antioxidant activity, participation in the signaling pathways, storage of docosahexaenoic acid, regulation of cell membranes. Plasmalogens abundantly present in the nerve tissue, in the heart, and in the lung surfactant. In this regard, the recession of their level is one of the indicators of neurodegenerative disorders and respiratory diseases. Plasmalogens biosynthesis de novo is well characterized, in general, however, metabolism of plasmalogens from the diet is still poorly described, despite their substantial presence in foods. It is the task of great importance to find sources and ways of bioavailability improvement of plasmalogens for their efficient recovery in associated diseases.
\end{abstract}

In this presentation are described and discussed:

1. The information on the structure and biological role of plasmalogens.

2. The data on the relationship between the level of plasmalogens and development of certain diseases.

3. The basic methods of nutritional regulation of plasmalogens level.

4. The main sources and methods for preparation of plasmalogens for pre-clinical and clinical studies.

The study was performed by a grant from the Russian Science Foundation (project No. 14-16-00055).

\section{Microwave-Assisted Extraction of Pectin from Pequi (Caryocar brasilense camb.) Fruit By-Product}

\author{
Leandro S. Oliveira ${ }^{1^{*}}$, Daniela P. Leão ${ }^{2}$, Bruno Botelho ${ }^{3}$ and Adriana S. Franca ${ }^{1}$ \\ ${ }^{1}$ DEMEC, Universidade Federal de Minas Gerais, Brazil \\ ${ }^{2} P P G C A$, Universidade Federal de Minas Gerais, Brazil \\ ${ }^{3}$ Centro Universitário de Belo Horizonte, Brazil
}

\begin{abstract}
Pectin, a polysaccharide found ubiquitously in cell walls of all plants, is a very important raw material for food and pharmaceutical products. Pectin extraction is a multi-stage physicochemical process, that involves hydrolysis and extraction of pectin macromolecules from plant tissue, purification of the liquid extract and isolation of the extracted pectin from the liquid. Conventional extraction techniques are time and solvent consuming, and also present a risk of thermal degradation of thermolabile components. Thus, microwave-assisted extraction can be a valuable tool in reducing processing time and degradation. In this study, a central composite rotational design was performed to assert the optimum conditions for microwaveassisted pectin extraction from powders prepared from the by-products of from pequi fruit, typically cultivated in the Brazilian Cerrado regions, and comprising $80 \%$ of the total fruit mass. The effects of microwave power, irradiation time and extraction temperature on the yield of pectin were evaluated. Pectin yields ranged from 12.75 to $20.79 \%$, for the powder obtained from the whole peel (fruit exocarp and mesocarp) and from 9.91 to $16.30 \%$ for the powder based only on mesorcarp. Results showed that temperature was the most important factor affecting pectin yield, with the best results being obtained at $108^{\circ} \mathrm{C}$ and $600 \mathrm{~W}$ microwave power. Regardless of the employed extraction conditions, pectins were deemed as high quality based on the values of their degree of esterification (proportion of methylated galacturonic acid groups to total galacturonic acid groups) that ranged from 51 to $80 \%$, with higher values obtained for the mesocarp-based powders.
\end{abstract}




\title{
Functional Efficacy of Food Components and Non-Nutrient Bioactives: A Case Study: Phaseolus vulgaris L.
}

\author{
Concettina La Motta ${ }^{1,2}$ \\ ${ }^{1}$ Department of Pharmacy, University of Pisa, Italy \\ ${ }^{2}$ Interdepartmental Research Center "Nutraceuticals and Food for Health" University of Pisa, Pisa, Italy
}

\begin{abstract}
Healthy diet is generally believed to have a major influence on both the prevention and development of chronic noncommunicable diseases like obesity, diabetes, cardiovascular events, cancer, and osteoporosis. Actually, most food-derived macro and micronutrients, as well as phytochemicals, can able to regulate key metabolic pathways and modulate oxidative stress and inflammatory signaling. Accordingly, interventions designed for healthy eating have become a hot topic in nutritional research and the intake of many food is nowadays highly recommended to get and maintain physical and mental health. The potential of pulses to address nutritional needs, for example, has been recently acknowledged by UN General Assembly, which declared 2016 International Year of Pulses. Although recognizing their important role in healthy diet, the full set of benefits that pulse crops can offer has not been fully characterized yet, and this results in an intriguing research opportunity. To raise awareness about the important role of pulses in healthy diets and their contribution to nutrition, we focused our attention on Phaseolus vulgaris L. (Zolfino Landraces), a small, pale yellow Tuscan bean coming from the very small mountainous region of Pratomagno, between the provinces of Arezzo and Florence.

This variety of bean differs from the other ones for the high content of flavonoids, known for their antioxidant and chelating properties. However, it also contains a many of amphiphilic compounds, represented by saponins, whose functional activities have not been thoroughly studied yet. Being characterized by a rigid hydrophobic group, connected via glycoside bonds to hydrophilic oligosaccharide chain, these compounds are acknowledged as natural surfactants able to exert beneficial effects on blood cholesterol levels. However, investigated in depth, saponins proved also to modulate the activity of aldose reductase, a key enzyme of glucose metabolism involved in long term diabetic complications, thus opening up a novel nutritional claim for this kind of pulse.
\end{abstract}

\section{Application of Dispersive Liquid-Liquid Microextraction to Analyses of Arsenic and Antibiotic Residues in Food Matrices}

\author{
Guoxin Lai ${ }^{1}$, Qiongqiong $\mathrm{Li}^{2}$ and Guoying $\mathrm{Chen}^{3 *}$ \\ ${ }^{1}$ Meizhouwan Vocational Technology College, China \\ ${ }^{2}$ Shanghai Institute for Food and Drug Control, China \\ ${ }^{3} U$.S. Department of Agriculture, Washington, DC, USA
}

\begin{abstract}
Dispersive liquid-liquid microextraction (DLLME) is an extraction technique with unique advantages such as simplicity, high throughput, large enrichment factor, minimal solvent usage, and low cost. Demonstrated in this work is application of this technique to analyses of inorganic arsenic (iAs) and antibiotic residues in food matrices. Successful examples include inorganic arsenic in fruit juices, oxytetracycline (OTC) in fish muscle, and fluoroquinolones (FQs) in swine liver. After extraction, DLLME was implemented by fast injection to establish a ternary emulsion system. The resulting tiny droplets promoted mass transfer enabling effective enrichment of target analytes. Quantification was performed by atomic fluorescence spectrometry (AFS) for iAs, or lanthanide-sensitized luminescence (LSL) for both OTC and FQ.
\end{abstract}

\section{Bioactive Compounds and Antioxidant Capacity of Green and Processed Leaves of Guayusa (Ilex paraguariensis Loes)}

\author{
Almudena García-Ruiz ${ }^{1}$, Nieves Baenas ${ }^{2}$, Ana M. Benítez-González ${ }^{3}$, Carla M. Stinco ${ }^{3}$, Antonio J. Meléndez-Martínez ${ }^{3}$, \\ Diego A. Moreno² and Jenny Ruales ${ }^{1}$ \\ ${ }^{1}$ Department of Food Science and Biotechnology, Escuela Politécnica National, Quito, Ecuador \\ ${ }^{2}$ Phytochemistry Lab. Department of Food Science and Technology, CEBAS-CSIC, Campus de Espinardo, Espinardo, Murcia, Spain \\ ${ }^{3}$ Food Colour E Quality Lab., Department of Nutrition E Food Science, Universidad de Sevilla, Facultad de Farmacia, Sevilla, Spain
}




\begin{abstract}
Ilex guayusa is an Amazonian evergreen tree of increasing interest due to their possible health benefits. This work aimed to provide a thorough description of the phenolic and carotenoid composition and antioxidant capacity of this plant, as well as to evaluate the effect of industrial processing on its composition and antioxidant capacity. A total of 14 phenolic compounds were identified and quantified. Chlorogenic acid and quercetin-3-O-glucoside were the main representatives of the hydroxycinnamic acids and flavonols, respectively. Five carotenoids were identified, showing lutein the highest concentration. DPPH and ORAC methods displayed a high antioxidant capacity. The industrial processing applied to the leaves modified the composition of bioactive compounds and antioxidant capacity of guayusa. In general, blanched guayusa retained the concentration of phenolic compounds and some carotenoids and similar antioxidant capacity as untreated green leaves. Oppositely, fermentation resulted in a negative and statistically significant $(\mathrm{p}<0.05)$ reduction and negatively reduced of the content of phenolics and the antioxidant capacity of guayusa. Therefore, blanched guayusa has potential for product development as functional ingredient in food industry.
\end{abstract}

\title{
Molecular Fingerprint of the Volatile Profile of the Aroma of Typical Italian Foods: Digital Database, Authenticity and Shelf Life
}

\section{Teresa Cecchi}

ITT Montani and Accademia delle Scienze, Italy

\begin{abstract}
Italy is well renowned for its specialty, traditional, and artisan foods. The evaluation of food quality, safety and authenticity requires a very deep knowledge of its composition. This is a very difficult challenge due to the great number of compounds that may be present. Our goal is to use the molecular signature of food aroma as a powerful authenticity, quality and shelf life criterion.
\end{abstract}

Head-space-solid-phase-micro-extraction, gas-chromatography-mass-pectrometry (HS-SPME-GC-MS) was used to determine volatile compounds of a number of typical Italian foods such as sourdough, sourdough beer, honey, chocolate, different kinds of pasta, clams; however, monocultivar extravirgin olive oils were the focus of our interest. In this case the importance of genotype and pedo-climatic conditions were also evaluated. This methodology is environmentally friendly because it avoids the use of solvents, it provides information on different classes of compounds simultaneously, it is fast, simple, and economical. The fibre we selected (DVB/CAR/PDMS) proved to be the most universal assembly for isolation of compounds with different physicochemical properties.

Common volatile constituents are many kinds of hydrocarbons, fatty acids, esters, aldehydes, ketones, alcohols, epoxides, furans, terpenes and terpenic derivatives, among others. Chemometry (principal component analysis and clustering) aims at assigning individual samples into groups based on their volatiles profiles.

The broad information provided by this methodology is useful not only for the construction of a digital database of the aroma of typical Italian foods but also for their nutritional value, oxidative stability, technological performance, quality, safety, botanical origin of vegetal samples, sample microbiota, and shelf life.

\section{Detection of Multiple Adulterants in Roasted and Ground Coffee by FTIR Employing Data Fusion}

\author{
Adriana S. Franca ${ }^{1^{*}}$, Nadia Reis ${ }^{2}$, Bruno Botelho ${ }^{3}$ and Leandro S. Oliveira ${ }^{1}$ \\ ${ }^{1} D E M E C$, Universidade Federal de Minas Gerais, Brazil \\ ${ }^{2}$ PPGCA, Universidade Federal de Minas Gerais, Brazil \\ ${ }^{3}$ Centro Universitário de Belo Horizonte, Brazil
}

\begin{abstract}
Over the last decades, the need for new and rapid analytical methods in the field of food analysis has prompted extensive research on Fourier Transform Infrared (FTIR) spectroscopy. FTIR methods are usually based on transmittance or reflectance readings, with reflectance-based methods being more commonly employed as routine methodologies for food analysis, and divided into Attenuated Total Reflectance (ATR) and Diffuse Reflectance (DR). ATR collects information from the sample surface whereas DR provides information from the entire sample, being a combination of internal and external reflection.
\end{abstract}


In this study, both methods are compared for discrimination of adulterants in roasted and ground coffee. Partial least square discriminant analysis (PLS-DA) hierarchical models were developed, with first level models providing discrimination between pure and adulterated coffee samples, and second level models being used to identify the specific adulterant. Data fusion (DF), the merging of analytical information provided by different sources (ATR and DR spectra) was evaluated as a tool for improvement of model accuracy. The evaluated adulterants were spent coffee grounds and roasted corn, barley and coffee husks. For the first level models, the use of DF decreased the percentage of misclassified samples in training/test sets from 19.6/14.7\% (DR) and 7.5/14.5\% (ATR) down to 2.5/4.5\%. The percentage of misclassified samples in the second level models was as low as $0 \%$ (DF spent coffee, training set). The proposed method is simple, fast, reliable for detecting adulteration in coffee samples, and capable of identifying these adulterants, even when in complex mixtures containing other adulterants.

\title{
Unveiling the Nature of Binding Between Selected Flavours to Canola and Pea Proteins and Effect of Chemical Modification of Proteins on Flavour Delivery
}

\author{
Kun Wang*and Susan D. Arntfield \\ Department of Food Science, University of Manitoba, Winnipeg, Canada
}

\begin{abstract}
In food systems, loss of free volatile compounds in the presence of proteins can greatly influence the quality and consumer acceptability of protein-containing foods. Interactions between selected flavours (aldehydes $\left(\mathrm{C}_{6-8}\right), \mathrm{ketones}\left(\mathrm{C}_{6-8}\right)$, hexyl acetate, benzaldehyde, dibutyl disulfide) and canola and pea protein isolates were studied using a GC/MS approach. The nature of binding was examined by monitoring protein thermal behavior in the presence of flavours using Differential Scanning Calorimetry (DSC); molecular forces were probed using various chemical reagents (urea, guanidine hydrochloride, propylene glycol, $\mathrm{Cl}_{3} \mathrm{CCOONa}, \mathrm{Na}_{2} \mathrm{SO}_{4}$, dithiothreitol). The extent of flavour binding was a function of protein source, protein isolation method and stereochemistry of the flavour compound. Interestingly, two volatile flavour by-products, 2-butyl-2octenal and 2-pentyl-2-nonenal, were detected from interactions between salt-extracted canola protein isolates and hexanal and heptanal, respectively, due to aldolisation reactions. The decreased denaturation enthalpy $(\Delta H)$ for DSC results inferred partial denaturation of proteins by these flavours. It was found that, compared to ketones, aldehyde flavours exhibited much higher "unfolding capacity" (lower $\Delta H$ ) than ketones, which may account for their remarkable binding affinities. By adding bond-disrupting agents, molecular forces responsible for the interactions were probed; hydrophobic interactions, hydrogen bond and ionic interactions were prominent for benzaldehyde, 2-octanone and hexyl acetate, whereas covalent interactions were implicated for octanal and dibutyl disulfide. Selectively modifying proteins via chemical (acetylation and succinylation) and enzymatic (Alcalase) approaches significantly altered protein-flavour binding affinities; this was influenced by the type of flavours and associated type of binding employed.
\end{abstract}

\section{Standoff Raman Technique for Rapid Detection of EMA in Olive Oil and Honey}

\author{
Anup Sharma*, Carlton Farley III, Aschalew Kassu, Jonathan Mills and Paul Ruffin \\ Alabama AE'M University, 4900 Meridian St N, Huntsville, AL, USA
}

\begin{abstract}
Pure extra virgin olive oil (EVOO) is mixed with cheaper edible oils and samples are kept inside clear glass containers, while a $785 \mathrm{~nm}$ Raman system is used to take measurements from a standoff distance of up to 1 meter. Several types of oils at various concentrations of adulteration are used. Ratios of peak intensities are used to analyze raw data, which allows for quick, easy, and accurate analysis. While conventional Raman measurements of EVOO may take as long as 2 min, all measurements reported here are for integration times of $5 \mathrm{~s}$. It is found that adulteration of EVOO with cheaper oils is detectable at concentrations as low as $5 \%$ for all oils used in this study. This is comparable with standard techniques, but only requires a fraction of the time to test the sample. Similarly, pure honey is mixed with high fructose corn syrup (HFCS) as well as rice malt syrup and kept inside clear glass containers. Integration time is $10 \mathrm{~s}$ for all samples in this part of the study, whereas the standard method for Raman analysis of honey samples takes about $2 \mathrm{~min}$. It is found that both HFCS and rice malt syrup are both easily detectable via this technique at concentrations as low as $20 \%$.
\end{abstract}




\title{
Improving Nutritional and Functional Properties of Pseudo-Cereals by Germination
}

\author{
Naofumi Morita ${ }^{1^{*}}$ and Pham Van Hung ${ }^{2}$ \\ ${ }^{1}$ Department of Food Packaging Technology, Toyo College of Food Technology, Japan \\ ${ }^{2}$ Department of Food Technology, Vietnam National University in HoChiMinh City, Vietnam
}

\begin{abstract}
Worldwide progress in milling and polishing apparatus and technology advances allows for most kinds of stable food, such as wheat or rice, to be refined by removing the germ, seed coat and aleurone layers. Consequently, these foods lose important nutrient ingredients and many people who eat these foods suffer from related illness. Germination is the start of life activity for grains, forming various low molecular bioactive and functional materials, and recent focus has been on germination of cereal grains.

Quinoa and amaranth were recommended to NASA as a potential 'new' crop for NASA's Controlled Ecological Life Support System. Quinoa seeds were germinated up to $72-\mathrm{h}$, and the $24-\mathrm{h}$ germinated quinoa flour substituted $10 \%$ into bread showed the largest loaf volume. Amaranth seeds were germinated at 20, 30 and $40{ }^{\circ} \mathrm{C}$ for 72 -h. After lyophilization and pulverization, bioactive and nutritional properties were tested. Amylase and protease activity were maximum after 24-h's germination. Changes in free amino-acids and protein compositions were tested during germination. Two allergenic bands disappeared out of three bands and increases in phytase activity, ferulic acid content, DPPH value, TEAC, phenolic compounds were observed. Further, the amount of soluble and insoluble dietary fiber increased significantly during the germination process. Buckwheat grains contain high amounts of flavonoid rutin which are beneficial to human health. The phenolics and flavonoid contents, flavonoid profile and antioxidant capacity of the extracts were examined during germination. As a result, the buckwheat grains germinated at 48-h were a good source of flavonoid compounds which can be used as functional foods.
\end{abstract}

\section{Beneficial Effects of a Diet with Walnuts in Alzheimer's Disease}

\author{
Abha Chauhan and Ved Chauhan \\ NYS Institute for Basic Research in Developmental Disabilities, Staten Island, NY, USA
}

\begin{abstract}
Amyloid beta-protein $(A \beta)$ is the major protein of amyloid deposits in the brain of patients with Alzheimer's disease (AD). Extensive evidence suggests neurotoxic effects of soluble oligomers of $A \beta$, and the role of oxidative stress and inflammation in AD. Walnuts are rich in components that have antioxidant and anti-inflammatory properties. Previous in vitro studies have shown that walnut extract inhibits $A \beta$ fibrillization, solubilizes its fibrils, and has protective effects against $A \beta$-induced oxidative stress and cell death. In the Tg2576 transgenic mouse model of AD (AD-tg), we have reported the beneficial effects of dietary supplementation of $6 \%$ (T6) or $9 \%$ walnuts (T9) [equivalent to 1 or 1.5 oz of walnuts per day in human] on the memory, anxiety and learning skills. The diets for the experimental and control mice were comparable as regards to total calories and the contents of protein, carbohydrate and fat. AD-tg mice on control diet without walnuts (T0) showed memory deficit, anxiety-related behavior, and severe impairment in spatial learning ability, position discrimination learning ability and motor coordination compared to the wild-type (Wt) mice on the same diet. AD-tg mice on diet with walnuts (T6, T9) showed a significant improvement in memory, learning skills, anxiety and motor development compared to AD-tg mice on diet without walnuts (T0). Recently, we have also analyzed A $\beta$ levels in the brain and blood samples of these mice at the ages of 4 months (before starting diet with walnuts), 14.5 and 19 months. At the age of 4 months, A $\beta$ levels (brain and blood) were similar in T0 and Wt mice. At the age of 14.5 and 19 months, T0 mice had significantly higher A $\beta$ levels than Wt mice. In the brain, T6 and T9 mice had significantly lower levels of soluble A $\beta$ oligomers compared to T0 mice. In the blood samples, the levels of A $\beta$ 1- 40 and 1- 42 were increased in T6 and T9 mice compared to T0 mice, suggesting that walnuts in the diet can increase the clearance of $A \beta$ from brain to the blood. These findings suggest that dietary supplementation with walnuts may have a beneficial effect in reducing the risk, delaying the onset, or slowing the progression of AD because it can (a) improve memory deficits and learning skills, (b) inhibit $A \beta$ fibrillization, $A \beta$-induced oxidative stress and $A \beta$-mediated cytotoxicity, and (c) reduce the levels of soluble $A \beta$ oligomers in the brain and increase ARegular clearance.
\end{abstract}




\title{
The Use of Saccharomyces cerevisiae Co-Products to Reduce Aflatoxin M1 in Milk
}

\author{
Carlos Corassin*, Bruna Gonçalves, Roice Rosim and Carlos Oliveira \\ Department of Food Engineering, School of Animal Science and Food Engineering, University of São Paulo, Pirassununga, SP, Brazil
}

\begin{abstract}
This study was to evaluate the effect of different sources of Saccharomyces cerevisiae (SC) biomass obtained from the sugarcane and beer industry on aflatoxin M1 (AFM1) excretion in milk from dairy cows receiving aflatoxin B1 (AFB1). The SC biomass (equivalent to $2.0 \times 1010 / \mathrm{g}$ ) consisted of inactive dry yeast sugarcane (DY), co-brewery partially dehydrated product (BY), cell wall $(\mathrm{CW})$ and autolyzed yeast (AY). AFB1 were administered to each intoxicated cow by daily oral bolus containing $480 \mu \mathrm{g}$ of the toxin. Twenty Holstein cows were assigned to free-stall and allowed free access to feed and water. A completely randomized design was used with 2 lactating cows assigned to each of 10 dietary treatments, as follows: negative controls (no AFB1 or SC biomass), positive controls (AFB1 alone), DY alone, DY + AFB1, BY alone and BY + AFB1, CW alone, CW + AFB1, AY alone and AY + AFB1. The intoxicated cows received AFB1 from day 1 to 7, while the SC biomass was administered with the AFB1 bolus from day 4 to 7 . Milk samples were collected daily and submitted to composition analyses and determination of AFM1 by ultra-performance liquid chromatography coupled to mass spectrometer. The reductions of AFM1 in milk by BY, DY, AY and $\mathrm{CW}$ at the end of the trial indicate a potential application of industrial fermentation by-products containing yeast cells in reducing the bioavailability of AFB1 in the diets of dairy cows and the excretion of AFM1 in milk.
\end{abstract}

\section{Feasibility of Post-Frying Vacuum Application in Improving the Performance of Frying Oil and Product Quality}

\author{
Azmil Haizam Ahmad Tarmizi ${ }^{1^{*}}$, Keshavan Niranjan ${ }^{2}$ and Michael Gordon ${ }^{2}$ \\ ${ }^{1}$ Malaysian Palm Oil Board, Malaysia \\ ${ }^{2}$ University of Reading, $U K$
}

\begin{abstract}
This paper discusses the application of vacuum during post-frying stage with the objectives to: (1) reduce the oil content in fried food; and (2) preserve the quality oil upon prolonged frying. Using potato chips as an illustrative product and palm olein as the frying medium, several protocols were investigated, either towards the end of frying process or post-frying stage. Lowering the pressure when the product was lifted from the oil gave a significant reduction in oil content. Vacuum drainage allows continuous water vapour evolution from the product - due to lower water saturation temperature - and thus hinders the surface oil from penetrating into the product structure. This is evidenced by the confocal laser scanning microscopy images which showed a visible boundary between the core and the crust regions. It is noted that oil distribution was only concentrated in the crust with insignificant oil residue in the core. Despite the product oil content can be lowered, the ability of oil to withstand high temperatures upon prolonged frying must also be taken into consideration. Interestingly, vacuum drainage significantly inhibited the formation of free fatty acid while the total oxidation value was slightly lowered, which in turn showed minimal reductions in the total colour difference value, viscosity and polar compounds. The feasibility of integrating moderate vacuum frying with high vacuum drainage in lowering the oil content in fried food was also examined. As expected, high vacuum drainage significantly lowered the rate of oil absorption irrespective to the vacuum frying conditions applied.
\end{abstract}

\section{Effect of Red Wine Pomace and Storage on Carcinogens Formation in Barbecued Beef Patties}

\section{Olga Viegas $^{1,2^{*}}$, Javier García-Lomillo ${ }^{3}$, Maria L. Gonzalez-SanJose ${ }^{3}$ and Isabel M.P.L.V.O. Ferreira ${ }^{1}$}

${ }^{1}$ LAQV/REQUIMTE, Laboratório de Bromatologia e Hidrologia, Departamento de Ciências Químicas, Faculdade de Farmácia, Universidade do Porto, Portugal

${ }^{2}$ Faculdade de Ciências da Nutrição e Alimentação, Universidade do Porto, Portugal

${ }^{3}$ Department of Biotechnology and Food Science, Faculty of Science, University of Burgos, Spain

\section{Abstract}

Polycyclic aromatic hydrocarbons (PAHs) and heterocyclic aromatic amines (HAs) were pointed out as the components of 
cooked meat with the highest carcinogenic potential. Since the mechanism of formation of these compounds may involves free radicals, the most promising mitigation strategies are the addition of natural products that can act as radical scavengers. Wine pomace is one by-products with a wide range of interesting compounds, presenting antioxidant and antimicrobial properties with preservative potential. This study aims to evaluate the influence of a red wine pomace seasoning (RWPS) and high-oxygen atmosphere storage on the formation of PAHs and HAs in barbecued beef patties.

Samples were analysed for PAHs and HAs contents using solid-phase extraction and high-performance liquid chromatography-diode array detection/fluorescence detection. In general, the levels of PAHs and HAs quantified in barbecued beef patties were low. The storage ( 9 days) promoted higher formation of PAHs in control patties without increase of HAs. Patties with RWPS cooked at preparation day presented higher levels of PAHs and HAs than control. Nevertheless, patties with RWPS cooked after storage presented significant lower levels of PAHs and HAs than control. Similar trend was observed for ABTS assay, which suggests a correlation between PAHs and HAs formation and the radical scavenging activity of meat. In conclusion, the addition of RWPS during patties storage at high-oxygen atmosphere increased the scavenging capacity and protected the endogenous antioxidants of meat, which contributed to a reduced formation of carcinogenic compounds during barbecuing.

\title{
Effects of Microcystin-Lr and Cylindrospermopsin on the Mineral Content of Lettuce Grown Hydroponically
}

\author{
Edgar Pinto ${ }^{1,2^{*}}$, Marisa Freitas ${ }^{2}$, Vítor Vasconcelos ${ }^{3}$ and Isabel M.P.L.V.O. Ferreira ${ }^{1}$ \\ ${ }^{1}$ Faculty of Pharmacy - University of Porto, Portugal \\ ${ }^{2}$ Health School - Polytechnic Institute of Porto, Portugal \\ ${ }^{3}$ Faculty of Sciences - University of Porto, Portugal
}

\begin{abstract}
Toxic cyanobacterial blooms in freshwaters constitutes nowadays a major ecological and human health problem worldwide. Anthropogenic eutrophication, resulting from increased input of nutrients, of surface waters leads to accelerated growth of cyanobacteria. Additionally, there is increasing evidence suggesting that recent changes in climate may also contribute to increase the episodes of cyanobacterial blooms [1,2]. Cyanobacteria can form a great variety of several secondary metabolites, with some of them identified as potent toxins (cyanotoxins). Microcystins are recognized as the most prevalent cyanotoxins in the environment and microcystin-LR (MC-LR), mainly produced by Microcystis aeruginosa, its predominant variant. However, cylindrospermopsin (CYN) has been gaining attention from the scientific community and public health authorities due to the invasive nature of its main producer, the cyanobacteria Cylindrospermopsis raciborskii [3]. Recent studies pointed out that irrigation water contaminated with cyanotoxins can produce toxic effects in crop plants [4]. Lettuce (Lactuca sativa L.) is an important commercial leafy vegetable that supplies essential minerals for human nutrition.
\end{abstract}

In this study, the effects of MC-LR and CYN at environmentally relevant concentrations of 1,10 and $100 \mu \mathrm{g} / \mathrm{L}$ were evaluated on the mineral content ( $\mathrm{Ca}, \mathrm{Mg}, \mathrm{K}, \mathrm{Na}, \mathrm{P}, \mathrm{Mn}, \mathrm{Fe}, \mathrm{Zn}, \mathrm{Cu}$ and $\mathrm{Mo}$ ) of Lettuce grown hydroponically.

Changes in the mineral content of Lettuce were observed at the end of 5 and 10 days after exposure to both cyanotoxins. In general, the mineral content of Lettuce decreased after exposure to MC-LR and increased after exposure to CYN, these effects being time and concentration-dependent.

\section{References}

1. Bláha L, Babica P, Maršálek B. 2009. Toxins produced in cyanobacterial water blooms - toxicity and risks. Interdiscip Toxicol 2: 36-41. doi: 10.2478/v10102-009-0006-2

2. Elliott JA.2012. Is the future blue-green? Are view of the current model predictions of how climate change could affect pelagic freshwater cyanobacteria. Water Res 46: 1364-1371. doi: 10.1016/j.watres.2011.12.018

3. Rzymski P,Poniedziałek B.2014. In search of environmental role of cylindrospermopsin: A review on global distribution and ecology of its producers. Water Res 66: 320-337. doi: 10.1016/j.watres.2014.08.029

4. McElhiney J, Lawton LA, Leifert C. 2001. Investigations into the inhibitory effects of microcystins on plant growth, and the toxicity of plant tissues following exposure. Toxicon 39: 1411-1420. doi: 10.1016/S0041-0101(01)00100-3 


\title{
Mitigation of Neo-Contaminant Formation in Chilean Bread: Effect of Phenolic Compounds from Tara (Caesalpinia spinosa) Extract
}

\author{
Franco Pedreschi ${ }^{1 *}$, Ilse Saavedra ${ }^{2}$, Andrea Bunger ${ }^{2}$, Rommy Zuñiga ${ }^{3}$, Romina Pedreschi ${ }^{4}$ and David Campos ${ }^{5}$ \\ ${ }^{1}$ Pontificia Universidad Católica de Chile/Department of Chemical Engineering and Bioprocess, Chile \\ ${ }^{2}$ Universidad de Chile/ Department of Food Science and Technology, Chile \\ ${ }^{3}$ Universidad Tecnológica Metropolitana/Department of Biotechnology, Chile \\ ${ }^{4}$ Pontificia Universidad Católica de Valparaíso/School of Agronomy, Chile \\ ${ }^{5}$ Department of Food Industry, Universidad Agraria La Molina, Lima, Perú
}

\begin{abstract}
Neo-formed contaminants such as acrylamide (AA) and furan have been detected in a wide variety of starchy foods, many of them are highly consumed in Chile. Chilean type bread called "hallulla" is one of the principal sources of furan and AA exposure to Chilean population. The objective of this research was to study the effect of a tara antioxidant extract over the formation of AA and furan in Chilean bread type "hallulla" without affecting negatively the final attractive sensory attributes for the consumers. Tara antioxidant extract (source of phenolic compounds) was obtained by lixiviation of tara pods with hot water at $60{ }^{\circ} \mathrm{C}$ as a solvent (liquid/solid: 60). Chemical characterization was done not only on the tara pods but also on the final antioxidant extract. In order to evaluate the effect of different concentrations of the phenolic compounds contained in the tara pod extract, acrylamide and furan concentration in bread pieces were detected and quantified by gas chromatography and mass spectrometry (GC-MS). Finally, sensory profile and quality of "hallulla" bread pieces were evaluated by a sensory panel formed by 11 assessors previously trained and validated. The tara pod extract contained total phenolics of $\sim 653 \mathrm{mg}$ tannic acid equivalents (EAT) for $\mathrm{g}$ of dry extract (quantified by Folin Ciocalteu Method) and presented an antioxidant capacity EC50 of $0,68 \mathrm{mg}$ EAT for $\mathrm{g}$ of dry extract according to the method of 2,2-difenil-1-picrilhidrazil radical (DPPH). AA and furan levels in "hallulla" pieces baked at $200{ }^{\circ} \mathrm{C}$ for 20 min was 82,7 and $1,9 \mathrm{ppb}$, respectively. When compared with control bread pieces (without phenolic antioxidant extract added), AA content was mitigated by $\sim 90 \%$ when $1500 \mathrm{ppm}$ of the phenolic tara pod extract was added to the bread pieces before baking while maintaining a similar descriptive profile and acceptable sensory quality of the final product. It is worth noting that the addition up to of $3000 \mathrm{ppm}$ of phenolic antioxidant tara pod extract did not diminish furan content more than that corresponding to the sample with $1500 \mathrm{ppm}$ of the phenolic tara pod extract. Furan formation was mitigated in $\sim 45 \%$ in bread pieces when the highest concentration of phenolic antioxidant tara extract tested was used.
\end{abstract}

\section{Activity of Natural Carotenoid Preparations against the Thermal Autoxidation of Oil- in-Water Emulsions}

\section{Sotirios Kiokias", Charikleia Dimakou and Vassiliki Oreopoulou}

Laboratory of Food Chemistry and Technology, School of Chemical Engineering, National Technical University of Athens, Polytechnioupoli Zografou, Iroon Polytechniou, Athens, Greece

\begin{abstract}
This research project has examined whether the addition of natural carotenoid preparations may influence the oxidative stability of sunflower oil-in-water emulsions. Focus is given on the use of certain natural mixtures (such as tomato, marigold, paprika and annatto extract), as their antioxidant activity has not been widely evaluated so far and therefore their mode of action needs further clarification, in particular in dispersed systems. The activity of natural carotenoid extracts against the emulsions autoxidative deterioration was estimated in terms of primary (lipid hydroperoxides) and secondary (off flavour volatiles) oxidation products. At a concentration of $2 \mathrm{~g} \mathrm{~L}^{-1}$ the tested carotenoids did not inhibit the production of hydroperoxides but they significantly retarded the formation of volatile aldehydes during storage at $30{ }^{\circ} \mathrm{C}$. In another series of experiments, several carotenoid extracts (paprika, annatto and marigold preparations), containing mainly polar carotenoids, added at an active concentration of $1 \mathrm{~g} \mathrm{~L}^{-1}$, exerted a strong activity against peroxide formation during the thermal accelerated oxidation $\left(60^{\circ} \mathrm{C}\right)$ of homogenised protein-based emulsions. On the contrary, carotene preparations rich in hydrophobic $\alpha, \beta$-carotenes and lycopene did not significantly differ than the control emulsion. Therefore, the carotenoid structure modulated its antioxidant activity, while concentration and emulsion structure may also affect carotenoid activity in protein dispersed systems. The understanding and optimisation of the carotenoid activity in novel emulsion systems, could offer the basis for their more systematic use by food industry as functional ingredients, which could protect the related food products from their sensory and nutritional oxidative deterioration.
\end{abstract}




\title{
Towards the Development of Bioactive Packaging
}

\author{
Zvi Hayouka and Tal Stern \\ Institute of Biochemistry, Food Science and Nutrition, The Robert H. Smith Faculty of Agriculture, Food and Environment, The Hebrew \\ University of Jerusalem, Rehovot, Israel
}

\begin{abstract}
Designing new approaches to inhibit microbial food contamination while maintaining quality, freshness, and safety are required. In my talk I will present our efforts towards the development of bioactive food processing surface technologies where the active agents are immobilized onto the surface materials via covalent linkages to prevent migration to the food. The active agents that we have developed are novel antimicrobial sequence random peptide mixtures. We have employed stepwise solidphase peptide synthesis and instead of using one pure amino acid at each coupling step, we used a mixture of two amino acids in a defined proportion. These cationic random peptides display strong antimicrobial activity towards food borne pathogens. Surface microbial attachment reveal to biofilm formation. Biofilm can be defined as structured aggregation of surface-attached microorganisms encased in an extracellular matrix. Bacterial cells within biofilms are much less susceptible to conventional antibiotics treatment than are bacterial cells in a planktonic state hence; it is very challenging to target them. According, to our findings we showed that our random peptide mixtures were able to prevent biofilm formation and more challenging even to eradicate mature biofilm. Our random peptides mixtures may be used as lead antimicrobial agents for many applications. We are currently developing chemical technologies to immobilize these random peptides mixture onto a model surface and characterizing their antimicrobial activity.
\end{abstract}

\section{Biocontrol of Staphylococcus aureus and Pectobacterium carotovorum on Fresh-Cut Produce by Treatment with Bacteriocins and Bacteriophages}

\author{
Eunjung Roh \\ National Institute of Agricultural Science, Republic of Korea
}

\begin{abstract}
The consumption of fresh-cut produce (ready-to-eat fruit and vegetables) has increased. As the consumption increases, problems were revealed. First, a certain amount of fresh-cut produce is deemed unusable by spoilage. Second, fresh-cut produce raise food safety concerns and a number of reports have referred to raw vegetables harboring potential food-borne pathogens. Staphylococcus aureus and Pectobacterium carotovorum were selected for major food-borne pathogen and spoilage bacteria which have been known to survive on fresh-cut produce under low temperature of the refrigerator. The staphycin and phage SP6 for S. aureus and carocin D and phage PP2 for P. carotovorum were used as biocontrol agents. In vitro, the bacteriocin and bacteriophage reduced viable cell number of target bacteria (S. aureus and P. carotovorum) by 3.0 to $6.0 \mathrm{log}$ units lower compared to the untreated control sample, respectively. The combination with bacteriocins and bacteriophages showed an increase in the reduction effect. We examined the effect of bacteriocins and bacteriophages via washing methods on artificially contaminated fresh-cut produce. On fresh-cut produce, they exhibited the same effect. Our research shows that washing application of bacteriocins and bacteriophages reduced the bacterial numbers at least as much as the general wash solution including organic acids. These applications with bacteriocins and bacteriophages may have possibilities for use in agriculture and food industry.
\end{abstract}

\section{The Characteristics of Alcohol Fermentation and Aroma Compounds in Winemaking Using Different Treatment Methods on Mulberry Juice}

\author{
Felix Narku Engmann ${ }^{*}$, Lizhi Yu², Jing Lin ${ }^{3}$ and Tian Baoming ${ }^{3}$
}

${ }^{1}$ Kumasi Polytechnic, Ghana

${ }^{2}$ Jiangsu University, PR China

${ }^{3}$ Southwest University, China

\begin{abstract}
The characteristics of alcohol fermentation of mulberry juice treated under four different conditions of ultra-high pressure (100 MPa/10 min, $200 \mathrm{MPa} / 10 \mathrm{~min}, 300 \mathrm{MPa} / 10 \mathrm{~min}, 400 \mathrm{MPa} / 10 \mathrm{~min}$ ), sulphur dioxide (60 mg/L), and a combination of
\end{abstract}


ultra-high pressure and sulphur dioxide $(30 \mathrm{mg} / \mathrm{L})$ were assessed in this work. The volatile aroma constituents of mulberry wine made from mulberry juice using different treatments were isolated by solid phase microextraction (SPME) and identified by gas chromatography-mass spectrometry (GC-MS). A total of twenty-five volatile aroma compounds of mulberry juice were identified, while nineteen volatile aroma compounds (five alcohols, four acids, and ten esters) were detected in mulberry wine. Ethyl acetate, butanoic acid ethyl ester, octanoic acid ethyl ester, acetic acid 2-phenylethyl ester, decanoic acid ethyl ester and ethyl 9-decenoate were the major esters and the main components of the mulberry wine. This was followed by 2-methyl, 1-propanol. Also, aldehydes formed the major volatile fraction in mulberry wine, followed by alcohols. High pressure processing maintained the original flavour distribution of the juice, as there were no significant differences $(p>0.05)$ among different mulberry wines in the types and concentrations of the volatile aroma components. Moreover, ultra-high pressure treatment $(300 \mathrm{MPa} / 10 \mathrm{~min}, 400 \mathrm{MPa} / 10 \mathrm{~min}$ ) drastic reduction of cfu to safe levels. It could be concluded treatment at $300 \mathrm{MPa} / 10 \mathrm{~min}$ and $400 \mathrm{MPa} / 10$ min could replace sulphur dioxide in winemaking in order to improve safety while maintaining the flavour compounds of mulberry wine.

\title{
Corn-Based Synbiotic Beverages Developed with Bifidobacterium Isolates from Dairy Beverages and Pharmaceutical Sources Identified Using a Multi-Gene Approach during Phylogenetic Analysis
}

\author{
Richard Nyanzi ${ }^{*}$, Piet J. Jooste ${ }^{1}$ and Jacobus N. Eloff ${ }^{2}$ \\ ${ }^{1}$ Tshwane University of Technology, South Africa \\ ${ }^{2}$ University of Pretoria, South Africa
}

\begin{abstract}
The 16S rRNA gene sequence analysis of Bifidobacterium species reveals high interspecies sequence similarity in the range of $87.7-99.5 \%$. This study aimed at illustrating the extent of superiority of a multigenic approach, involving proteincoding genes, in comparison to the $16 \mathrm{~S}$ rRNA gene, to precisely delineate presumptive Bifidobacterium isolates obtained from probiotic milk beverages, culture collections and pharmaceutical probiotic preparations. The growth and viability of precisely identified Bifidobacterium isolates was optimized in corn-based synbiotic beverages as an alternative to the dairy-based probiotic beverages which may not be suitable due to lactose intolerance or religious reasons. Oligonucleotide pairs PurF-rev/PurF-uni; RpoC-uni/RpoC-rev; DnaB-uni/DnaB-rev; DnaG-uni/DnaG-rev; and ClpC-uni/ClpC-rev amplified housekeeping genes while $27 \mathrm{~F} / 1492 \mathrm{R}$ amplified the $16 \mathrm{~S}$ rRNA gene of the presumptive bifidobacteria in a polymerase chain reaction. Sequences of $16 \mathrm{~S}$ rRNA gene and some protein-coding genes effectively identified the isolates. Phylogenetic analysis together with concatenation showed that clpC, purF and DnaG genes had over 8-fold better discriminatory power than the 16S rRNA gene in discriminating between Bifidobacterium isolates. However, phylogenetic analysis involving DnaB and RpoC gene sequences or their concatenated trees showed discrepancies in clustering isolates with designated type strains. The study revealed approaches which sustained growth and viability of selected Bifidobacterium strains in corn-based non-dairy synbiotic beverages. The population levels were well above the recommended therapeutic minimum of $1 \times 107$ colony forming units per milliliter $(\mathrm{cfu} / \mathrm{mL})$ during the shelflife period.
\end{abstract}

\section{Optimization of Osmotic Dehydration of Bananas Slices in Sucrose Solutions Using Response Surface Methodology}

\author{
Ali Ferradji*, Belhachat D and FS Ait Chaouche \\ High National School of Agronomy, Department of Food Sciences, Algeria
}

\begin{abstract}
Osmotic dehydration of bananas slices in continuous kinetic was studied using sugar solution at different concentration (30-74), temperature $\left(40-60{ }^{\circ} \mathrm{C}\right)$ and time (60-120 $\left.\mathrm{min}\right)$. The response surface methodology was used to optimize the effects of temperature, sucrose concentration and immersion time. A Composite Central Design (CCD) was used with weight loss (WL\%), moisture loss (WL\%) and solid gain (GS\%) as responses. Moisture sorption isotherms of the bananas slices were determined at 25 and $45^{\circ} \mathrm{C}$ by balancing the atmosphere with relative humidity fixed by dilute solutions of sulphuric acid. The models obtained for all the responses were significant $(\mathrm{P}<0.05)$. The results suggest that WL and SG can reach 55.65\%, and $5.47 \%$ respectively after $90 \mathrm{~min}$ of the osmotic dehydration. The GAB equation provided a good fit to experimental data $(<6 \%$ RMS). Monolayer moisture contents for date slices bananas was $9.916 \%$ and $7.95 \%$ respectively at 25 and $45^{\circ} \mathrm{C}$. The enthalpy
\end{abstract}


of sorption of the monolayer at $25^{\circ} \mathrm{C}$ was $44.01 \mathrm{KJ} /$ mole. Heat of desorption estimated using the Clausius-Clapeyron equation was 38.85 kcal.mol ${ }^{-1}$.

\title{
Encapsulation of Active Compounds for Increasing Shelf Life of Fresh Products
}

\author{
Silvia Matiacevich*, Katherine Alarcón, Natalia Riquelme and Carla Arancibia \\ Food Properties Research Group, Department of Food Science and Technology, University of Santiago de Chile, Chile
}

\begin{abstract}
Synthetic antimicrobial chemicals have been utilized for decades to control microbial growth. However, the study of functional and active natural ingredients has been increasing because modern consumers are demanding fresh, safe and healthy natural foods. This area is within the top ten for food innovation. The activity of essential oils and their molecular constituents as antimicrobials agents has been widely studied against many microorganisms, including several pathogens. But due to their volatile and lipofilic characteristics is necessary to protect them when is incorporating to hydrophilic food matrix. The advantages of micro and nanoencapsulation have opened up new opportunities by improving their shelf-life as a result of the incorporation of an active (antimicrobial and antioxidant) ingredient. The encapsulation of antimicrobial agents (essential oils and its main component) in an alginate matrix were studied in edible films [1,2] and as freeze-dried ingredients. The effect of antimicrobial agents depended on storage conditions and encapsulating agents used [1,3]. Besides, an increasing shelf life of fresh products (chicken breast and carrots) were observed when active compounds encapsulated were applied as edible coating [4].
\end{abstract}

\section{References}

1. Navarro, Arancibia, Herrera, Matiacevich. 2016. Effect of type of encapsulating agent on physical properties of edible films based on alginate and thyme oil. Food Biop Proc 97: 63-75. doi: 10.1016/j.fbp.2015.11.001

2. Bustos, Alberti, Matiacevich. 2016. Edible antimicrobial films based on microencapsulated lemongrass oil. J Food Sci Tech 53(10): 832-839. doi: 10.1007/s13197-015-2027-5

3. Matiacevich, Riquelme, Herrera. 2015. Conditions to prolonged release of microencapsulated carvacrol on alginate films as affected by emulsifier type and pH. Int J Pol Sci 2015: 173193. doi: 10.1155/2015/173193

4. Matiacevich, Acevedo, López. 2015. Characterization of edible active coating based on alginate-thyme oil-propionic acid for the preservation of fresh chicken breast fillets. J Food Proc Pres 39(6): 2792-2801. doi: 10.1111/jfpp.12530

\section{A Clear View on Transparency: and How it Builds Consumer Trust}

\author{
JJ. Jones* and Charlie Arnot \\ The Center For Food Integrity, 2900 NE Brooktree Ln \#200, Kansas City, MO, USA
}

\begin{abstract}
It's simple: If you increase transparency, you will increase trust. Earning consumer trust is critical for those involved in today's food system. The reality that our food is safer than ever before - and remarkably affordable - can be overshadowed by a lack of trust. So, how do producers, food companies and restaurants earn trust? What do consumers want from them? The answer, in part, is transparency. The Center for Food Integrity's (CFI) 2015 consumer trust research proves that as those in the food system increase transparency, they will also increase consumer trust. The link between transparency and trust is real, direct and powerful. But, transparency can be an elusive term, so CFI set out to define it. The research helps the food system better understand what it takes to earn and maintain consumer trust.
\end{abstract}

Three objectives for the session:

1. Establish understanding that large food companies are susceptible to public sentiment that profit is being placed ahead of public interest, undermining consumer trust.

2. Attendees understand the value of increasing transparency and the related impact on trust in their products, processes, people and brands.

3. Attendees have an understanding of and appreciation for best practices in transparency that they can apply in their company. 


\title{
Enzymatic Synthesis of Amphiphilic Acyl Ascorbate and its Suppressive Effect on Lipid Oxidation
}

\author{
Yoshiyuki Watanabe
}

Kindai University, Japan

\begin{abstract}
Lipid oxidation can lead to the deterioration of quality in food. Ascorbic acid is well known as a water soluble vitamin with high reductivity; therefore, it is widely used as an antioxidant in foods and cosmetics. Acyl ascorbate was synthesized through the condensation of ascorbic acid with a fatty acid using a lipase in a water miscible organic solvent. Since acyl ascorbate consists of an ascorbyl part as a hydrophilic group and an acyl residue as a hydrophobic group, it may be an edible emulsifier with both surface activity and reductivity. Enzymatic synthesis of acyl ascorbate is more advantageous than using a chemical method because of the simplicity of its reaction process and high regioselectivity. Continuous production can be achieved by using a continuous stirred tank reactor (CSTR) or a plug flow reactor (PFR) packed immobilized lipase. The long term operational stability of the immobilized lipase was examined for various acyl ascorbates. The productivity for the PFR was much higher than that for the CSTR. The immobilized lipase was found to be stable, and the reactor was steadily operated for at least 11 days. The oxidation processes were kinetically analyzed for linoleic acid in the presence of ascorbic acid or acyl ascorbate. At any given temperature, the rate constant for the oxidation with no additive was higher than that with the addition of ascorbates. There was a tendency for the constant, in the presence of ascorbate with a larger acyl chain length, to be slightly smaller.
\end{abstract}

\section{Matrix Effects on Release of Strawberry Favour Volatiles from Tannia (Xanthosoma sagittifolium) Gels}

\author{
Patiricia G. Owusu-Darko' $^{1^{*}}$, Alistair Paterson ${ }^{2}$ and John R. Piggott ${ }^{2}$ \\ ${ }^{1}$ Kumasi Technical University, Ghana, West Africa \\ ${ }^{2}$ University of Strathclyde, Scotland, UK
}

\begin{abstract}
Tannia (Xanthosoama saggitifolium) is an underutilized tropical tuber crop with potential for use as industrial starch for the food industry. Flavour release from dairy gels prepared from starch and pulp from red tannia was studied. One concentration of strawberry flavour was used with two levels of tannia starch or pulp and sucrose and presence or absence of $\kappa$-carrageenan; HS-SPME-GC showed that individual components behaved differently in the varying gel matrices. Headspace concentrations of flavour components were highly significantly $(\mathrm{p}<0.001)$ different between gels; an exception was ethyl iso pentanoate ( $\mathrm{p}>0.05)$. Significant interactions were observed between sucrose and $\kappa$-carrageenan. Principal component analysis (PCA) generated a two-factor space, with 3 clusters 1- ethyl butyrate on PC1 linked to one, ethyl hexanoate on PC2 linked to a second. A third cluster was linked to styralyl acetate, cis hexenyl acetate, and beta ionone. Interactions between experimental factors were the main driving force on PLS Factor 1. Compositional factors - starch type whether - whether extracted starch or pulp (ST) and starch level (SL), sucrose level (SGL) and presence or absence of $\kappa$-carrageenan (CL) influenced score on factor 2. Absence of $\kappa$-carrageenan in high starch gels showed similarity in flavour release patterns to presence of $\kappa$ carrageenan in low starch matrices.
\end{abstract}

\section{Young Researchers Forum}

\section{Green Sustainable Supercritical Carbon Dioxide for Enzymatic Synthesis of Diacylglycerols and Monoacylglycerols}

\author{
Nazanin Vafaei ${ }^{1,2^{*}}$, Martin G. Scanlon ${ }^{1}$, Curtis B. Rempel ${ }^{1,3}$, Peter J.H. Jones ${ }^{1,2}$ and Michael N.A. Eskin ${ }^{1}$ \\ ${ }^{1}$ University of Manitoba, Canada \\ ${ }^{2}$ Richardson Centre for Functional Foods and Nutraceuticals (RCFFN), Canada \\ ${ }^{3}$ Canola Council of Canada, Canada
}




\title{
Abstract
}

A shift to sustainable practices is urgent for today's world. Supercritical carbon dioxide $\left(\mathrm{SC}-\mathrm{CO}_{2}\right)$ technology uses green sustainable chemistry routes to synthesise new materials. Applying the $\mathrm{SC}-\mathrm{CO}_{2}$ as an environmentally friendly technology for producing diacylglycerol (DAG) and monoacylglycerol (MAG) is the focus of this study. DAG-enriched oils were introduced because of their beneficial effect in reducing visceral fat. MAGs are the most commonly used emulsifiers in the food industry. A SC- $\mathrm{CO}_{2}$ system was developed for the enzymatic alcoholysis of MAG and DAG-enriched oil from soybean oil in order to exclude the use of organic solvents. The reaction for DAG-enriched oils production was conducted under $\mathrm{SC}-\mathrm{CO}_{2}$ and/or atmospheric conditions at $60{ }^{\circ} \mathrm{C}, 75 \mathrm{bar}$ using a glycerol/soybean oil at a molar ratio of $1: 2$ and novozyme 435 lipase. However, MAGs were produced only under $\mathrm{SC}-\mathrm{CO}_{2}$ condition at $70{ }^{\circ} \mathrm{C}, 276$ bar using 1,2 -propanediol/soybean oil at a molar ratio of 10:1 with and without novozyme 435. Two time course studies of the enzymatic reaction for 1, 3, 4 and 8 hours for DAG-enriched oils and for 2, 4, 5 and $6 \mathrm{~h}$ for MAGs were conducted. Nuclear magnetic resonance (NMR), specifically 31P-NMR, was used to determine the composition of the MAGs and DAG-enriched oils. A much higher yield of total DAG (38\%), specifically 1,3-DAG, was obtained under SC- $\mathrm{CO}_{2}$ conditions compared to the reaction conducted under atmospheric conditions (5.5\%). Under the enzymatic reaction for MAG synthesis, the highest yield for total MAG was, $26 \%$ after $4 \mathrm{~h}$, while this number was $0.18 \%$ for the non-enzymatic reaction.

\section{Metabolomic Profiling and Enzyme Assisted Extraction of Bioactive Compounds from Kakadu Plum (Terminalia ferdinandiana) - A Native Plant of Australia}

\author{
Mridusmita Chaliha ${ }^{1}$, David Williams ${ }^{2}$, Heather. Smyth ${ }^{1}$, Sharon Pun ${ }^{2}$, David Edwards ${ }^{2}$ and Yasmina Sultanbawa ${ }^{1}$ \\ ${ }^{1}$ Queensland Alliance for Agriculture and Food Innovation (QAAFI), The University of Queensland, Queensland, Australia \\ ${ }^{2}$ Department of Agriculture and Fisheries (DAF), Queensland, Australia
}

\begin{abstract}
Kakadu plum (KP, Terminalia ferdinandiana) is a traditional fruit that has been consumed for its nutritional and therapeutic value by indigenous Australians for thousands of years. Presence of high levels of ellagic acid (EA) and ascorbic acid (AA) makes it a rich source of antioxidants. Non-targeted metabolomic profiling of polar extracts of the KP fruit, seeds and leaves identified 158 metabolites including amino acids, organic acids, phenolic acids, esters and sugars. In addition to detecting AA and gallic acid, previously unreported metabolites such as galacturonic acid, rhamnose, xylitol, xylulose, maltose, myo-inositol and palatinose were tentatively identified. Presence of these sugars indicated the presence of pectin in KP tissue. Therefore, chemical extraction and subsequent characterization was carried out on the KP pectin. The extracted pectin (yield 85\%) had an equivalent weight of $251 \mathrm{~g}, 13 \%$ methoxyl content and 40\% degree of esterification. Non-polar extracts from flesh, seeds and leaf tissues showed the presence of saturated and unsaturated fatty acids, including lauric acid, palmitic acid, steric acid, linolenic acid and oleic acid. To obtain enhanced release and recovery of bio-active compounds like EA and AA, that are known to get trapped within plant cells, enzymatic assisted extraction of bioactive compounds from KP tissue was carried out with pectinase using a central composite rotatable design (CCRD). The resultant 20 extracts showed high concentration of EA ranging from $51-256 \mathrm{mg} / \mathrm{ml}$ and AA ranging from $67-121 \mathrm{mg} / \mathrm{ml}$ at alkaline and acidic $\mathrm{pH}$ respectively. The total phenolic activity and antimicrobial effects of the extracts were investigated.
\end{abstract}

\section{Tracing the Pathway of Oxidation Using Hydrophobic and Hydrophilic Natural Antioxidants in an Oil in Water Emulsion Food System}

\author{
Sara Ghorbani Gorji ${ }^{*}$, Heather E. Smyth ${ }^{2}$ and Melissa Fitzgerald ${ }^{1}$ \\ ${ }^{1}$ The University of Queensland, School of Agriculture and Food Science, Australia \\ ${ }^{2}$ The University of Queensland, Queensland Alliance for Agriculture and Food Innovation (QAAFI), Australia
}

\begin{abstract}
Oxidative instability of oil in water emulsion $(\mathrm{O} / \mathrm{W})$ food systems reduces their shelf life. Usually synthetic antioxidants are added to the $\mathrm{O} / \mathrm{W}$ emulsion to prevent the development of rancidity. The objective of this work was to first elucidate the kinetic of oxidation in an $\mathrm{O} / \mathrm{W}$ emulsion system stored for 92 days at $4{ }^{\circ} \mathrm{C}, 25{ }^{\circ} \mathrm{C}$ and $38{ }^{\circ} \mathrm{C}$. Secondly, keeping in view the increasing demand for natural antioxidants, to investigate the antioxidant efficacy of hydrophobic and hydrophilic natural antioxidants in an $\mathrm{O} / \mathrm{W}$ emulsion system stored at $38^{\circ} \mathrm{C}$ for 60 days. Due to the complexity of the headspace of degraded oils, comprehensive two-dimensional gas chromatography (GC x GC) coupled with mass spectrometry (MS) was the technique
\end{abstract}


of choice, providing four dimensions of separation. Descriptive sensory analysis of samples, combined with metabolomics provided new insights about the efficacy of both natural hydrophobic and hydrophilic antioxidants in an $\mathrm{O} / \mathrm{W}$ emulsion system. The evolution of oxidation products was analyzed during storage, and very close relationships were found between the volatile compounds generated from fatty acids and the original oil composition in the fatty acids. Among more than 80 compounds, 4-ethylcyclohexan-1-ol, 4-Methyl-cyclohexan-1-ol, 3-hexenal, Heptan-2-one, benzeneacetaldehyde, 3-cyclohexene-1carboxaldehyde, butanal, 2-methyl, benzene, 1-methyl-3-(1-methylethyl), 1-octen-3-one, 2,4-heptadienal, 1,3-octadiene and decane, 2,3,5,8-tetramethyl were detected in high levels of oxidation. Taking these data into account the ability of hydrophobic and hydrophilic antioxidants to reduce the concentration of these compounds was studied.

\title{
Deacidification of Cranberry Juice by Electrodialysis: Impact of Membrane Types and Configurations on Acid Migration and Juice Physicochemical Characteristics
}

\author{
Elodie Serre $^{1,2^{*}}$, Elodie Rozoy ${ }^{1,2}$, Karine Pedneault ${ }^{1,3}$, Stella Lacour $^{4}$ and Laurent Bazinet $^{1,2}$ \\ ${ }^{1}$ Institute of Nutrition and Functional Foods (INAF), Université Laval, Quebec (QC), Canada \\ ${ }^{2}$ Department of Food Sciences, Pavillon Paul Comtois, Université Laval, Quebec (QC), Canada \\ ${ }^{3}$ Quebec Agrifood Development Center (CDBQ), Sainte-Anne-de-La Pocatière (QC), Canada \\ ${ }^{4}$ European Membrane Institute (IEM), University of Montpellier, Place Eugène Bataillon, Montpellier, France
}

\begin{abstract}
Cranberry is a typical fruit from North America having a very high acidity that makes raw juice hardly acceptable for consumers. In this study, the reduction of juice acidity was investigated using electrodialysis (ED) with different types of membranes and cell configurations. Electrodialysis is an ecofriendly membrane technology used in a large range of food applications. Three different ED configurations were tested at the laboratory scale: bipolar and anion-exchange membranes (ED2MB), bipolar and ultrafiltration membranes (ED2MBUF), and cation-exchange and ultrafiltration membranes (EDUF). Each configuration was evaluated in terms of juice physicochemical parameters (titratable acidity, conductivity, total soluble solids, color, anthocyanins, organic acids and mineral contents) and electrodialytic parameters (membrane conductivity and thicknesses, global system resistance). In ED2MB configuration, a 40\% deacidification rate was reached after 3 hours of treatment $(80 \%$ after $6 \mathrm{~h}$ ) whereas $0 \%$ and only $8 \%$ were obtained after 3 hours with ED2MBUF and EDUF configurations, respectively. Furthermore, a selective migration of organic acids was observed with the ED2MB configuration: citric acid (22 $\mathrm{ppm} / \mathrm{min})$, malic acid (11 ppm/min). Consequently, ED2MB configuration allows the deacidification of cranberry juice and the production of pure acids (no waste generated) without any chemical consumption due to the bipolar membrane in-situ generation of proton and hydroxyl species from water.
\end{abstract}

\section{Essential Oil Based Nanoformulation for Pest Control in Grain Storage System - A Step Towards Food Safety}

\author{
Prachi Singh", Santosh Satya and S.N. Naik \\ Centre for Rural Development and Technology, Indian Institute of Technology, New Delhi, India
}

\begin{abstract}
Stored grains are susceptible to insect- pest attack which causes undesirable changes in quantitative as well as qualitative properties of grains. Currently, agrochemicals are being used indiscriminately to salvage grains but they contaminate the food chain posing a threat to food safety. Therefore, intensive research centered at exploring safer and natural alternatives is going on. One possible approach adopted by a few researchers is the development of essential oils based nanoformulation that has tremendous application in food sector especially in post harvest processing.
\end{abstract}

The present paper aims at development and characterization of 5\% Mentha arvensisis essential oil based nanoemulsion (oil-in-water) incorporated with $1 \%$ aqueous filtrate of Neem and Karanja de-oiled cake. The emulsion was tested for the stability of encapsulated bioactive compound by Headspace GC-MS technique (pre and post formulation) and ATR-FTIR technique. Droplet size distribution of prepared formulation was measured by DLS and the average size was found to be 36.61 nm. Morphological observation of sample under Scanning Electron Microscopy (SEM) depicts spherical shape of the droplets which is instrumental in enhancing the release performance and bioavailability of active ingredient. The bio-efficacy of above developed formulation has been tested against $R$. dominica - (a ubiquitous pest of wheat) by filter paper impregnation method. It was observed that the contact toxicity activity of emulsion produced $86 \%$ mortality of $R$. dominica adults at a dose of 31.25 $\mathrm{mgL}^{-1} \mathrm{~cm}^{-1}$. 
Research findings reveal that nanoemulsion prepared from Mentha oil with synergistic combination of de-oiled cakes may prove as a green alternative for insect-pest management in small scale grain storage structures/ seed storage system. For field applications, understanding of release kinetics for the availability of adequate quantity of bioactive constituent (Menthol) over storage time has to be ensured.

\title{
Validation of Analysis Method and Monitoring on Organic Acids in Liquors
}

\author{
Jong-Min Park* and Ki-Teak Lee \\ Department of Food Science and Technology, Chungnam National University, Daejeon, South Korea
}

\begin{abstract}
In this study, three different HPLC methods were used for separation and quantification of organic acids (oxalic, tartaric, malic, lactic, acetic, citric, and succinic acids). Method 1 was performed by UV detector (210 nm) using a $50 \mathrm{nM} \mathrm{KH} \mathrm{PO} \mathrm{buffer}$ ( $\mathrm{pH}$ 2.8) as a mobile phase and two columns (Waters Nova-pak C18 and Hypersil GOLD aQcolumns). The detection order was oxalic, tartaric, malic, lactic, citric, and succinic acids. Method 2 and 3 were carried out at post-column system using a 3 $\mathrm{mM} \mathrm{HClO}$ mixed with BTB solution and UV detector $(440 \mathrm{~nm})$. Two columns of Hypersil GOLD aQ and SUPLECOGEL C-610H were used for method 2, resulting in detection order of oxalic, tartaric + citric (co-elution), malic, succinic, lactic, and acetic acids. In method 3, SUPLECOGEL C-610H column was used for separation of organic acids (oxalic, tartaric + citric (co-elution), malic, succinic, lactic, and acetic acids). Organic acids profiled with seven species of liquor (beer, Takju, red wine, white wine, whiskey, rice wine (cheongju), distilled soju). The most abundant acid in beer was lactic acid. Rang of lactic acid in Method 2 and Method 3 were $48.5-182.5 \mathrm{mg} / \mathrm{L}$ and $188.8-835.8 \mathrm{mg} / \mathrm{L}$, respectively. The most organic acid in wine was tartaric acid, range of tartaric in method 1 and 2 were $1507.6-2397.2 \mathrm{mg} / \mathrm{L}$ and $1160.8-2652.4 \mathrm{mg} / \mathrm{L}$. Whiskey had only acetic acid. Amount of acetic acid in method 1 and 3 were 788.7 and $168.6 \mathrm{mg} / \mathrm{L}$. In distilled soju, organic acid was not detected.
\end{abstract}

\section{Validation of Analysis Method and Monitoring on Diacetyl in Liquors}

\author{
Hyeon-Hwa Lee, Jung-Ah Shin and Ki-Teak Lee \\ Department of Food Science and Technology, Chungnam National University, Daejeon, South Korea
}

\begin{abstract}
Diacetyl is a natural byproduct of fermentation and known to be an important flavor compound in liquors. Meanwhile, diacetyl exceeded the standard level (over $1 \mathrm{ppm}$ ) has an undesirable effect on liquor flavor. The first aim of this paper is to validate a high performance liquid chromatography method for the quantification of diacetyl in liquor based on the derivatization reaction of diacetyl with 1,2-diaminobenzene (OPDA). Some of the liquor was pretreated (filtering and centrifugation) owing to their physical properties. The derivatization was performed with OPDA in $\mathrm{pH} 8.0$ at $60{ }^{\circ} \mathrm{C}$ for 3 hour for improving the sensitivity in the detector. After the derivation, diacetyl was detected by ultraviolet detector at $313 \mathrm{~nm}$. The results showed that the Coefficient of Correlation for external standard curve was 1 in the range of $0.05-10 \mathrm{ppm}$ and the limit of quantitation (LOQ) was $0.039 \mathrm{ppm}$. The second aim is to monitor the diacetyl content for quality control on liquors consumed in Korea. Various types of liquor were selected such as 39 Yakju, 72 Takju, 24 Wine, 12 Whiskey, 9 Distilled Soju, 38 General Distilled Spirits, 15 Diluted Soju, 10 Brandy, 25 Liqueur, 31 Fruit Wine, 33 Others, 76 Beer, and 15 Cheongju. The content of diacetyl in liquor ranged from 0.005 to $3.655 \mathrm{ppm}$. The highest content of diacetyl was found from Fruit Wine $(0.432 \pm 0.767 \mathrm{ppm})$. In contrast, Brandy has the lowest content of diacetyl $(0.017 \pm 0.012 \mathrm{ppm})$. Different type of liquors did not show distinct difference on diacetyl content.
\end{abstract}

\section{Are Glucose and Surfactant Essential Nutrients for the Production of Biomass and Bacteriocin in Lactobacillus plantarum B21?}

Elvina Parlindungan*, Khanh Tran, Harsharn Gill and Bee May

RMIT University, 124 La Trobe St, Melbourne VIC, Australia

\section{Abstract}

Lactobacillus plantarum is classified as safe (GRAS) and has been used in a number of industrial and food applications. 
Lactobacillus plantarum B21, isolated from traditional Vietnamese fermented pork, has been reported to be a strong acid and bacteriocin producing strain with broad inhibitory spectra towards LAB and pathogenic bacteria. It is well documented that the response of Lactic Acid Bacteria (LAB) to growth medium and conditions is strain specific. In particular, glucose was found to be the key carbohydrate source for a wide range of LAB strains and Tween 80 an essential ingredient for bacteriocin production. Therefore, the aim of this study was to investigate the effect of glucose and Tween 80 on biomass and bacteriocin production of Lactobacillus plantarum B21. It was found that Tween 80 has significant positive effect on bacteriocin production but has little or no effect on biomass production. On the contrary, glucose has little or no effect on bacteriocin production but is essential for biomass production. In addition, we found that high temperatures of $37{ }^{\circ} \mathrm{C}$ and $42{ }^{\circ} \mathrm{C}$ resulted in significantly higher bacteriocin production by this specific strain. These results are in contrast to what was reported in numerous earlier studies. These results clearly demonstrate that nutrient requirements are strain specific. Further studies are being carried out to understand the molecular mechanisms of Tween 80 and glucose in relation to biomass and bacteriocin production in L. plantarum B21.

\title{
Antioxidant Activity, Total Phenolic Content and Physicochemical Properties of Carbonated Erica arborea Herbal Tea Beverage
}

\author{
Gülşah OZCAN SINIR', Senem SUNA, Canan Ece TAMER, Bige İNCEDAYI and Ömer Utku ÇOPUR \\ Uludag University, Agriculture Faculty, Department of Food Engineering, Görükle, Bursa, Turkey
}

\begin{abstract}
The aim of this study was to use Erica arborea water extract for developing a novel herbal tea beverage. For this reason, 10 $\mathrm{g}$ dried Erica arborea leaves were infused with $1 \mathrm{~L}$ boiling water $\left(100^{\circ} \mathrm{C}\right)$ for $5 \mathrm{~min}$. Then herbal tea extract was adjusted to $8^{\circ}$ brix with the addition of sucrose, organic acids (citric acid and ascorbic acid) and natural lemon flavor. Antimicrobial agents (100 mg/L Na-benzoate and $190 \mathrm{mg} / \mathrm{L} \mathrm{K}$-sorbate) were added to beverage for maintaining food safety. After the mixing of ingredients, beverage was carbonated with 4.5 units $\mathrm{CO}^{2}$. The water soluble dry matter, $\mathrm{pH}$, color $\left(L^{*}, a^{*}, b^{*}\right)$ and turbidity (NTU) values were analyzed in beverages. In the view of estimating the functionality, extractable and bioaccessible phenolics and antioxidant activities of chemical and physiological extracts with DPPH, FRAP and CUPRAC methods and nutritional parameters such as ascorbic acid and $\mathrm{Fe}, \mathrm{Ca}, \mathrm{Mg}, \mathrm{K}, \mathrm{Na}$ minerals were assessed. The extractable and bioaccessible phenolics of the beverage were found as $174.06 \pm 24.53 \mathrm{mg} \mathrm{GAE} 100 \mathrm{~mL}^{-1}$ and $96.07 \pm 3.96 \mathrm{mg} \mathrm{GAE} 100 \mathrm{~mL}^{-1}$, respectively. Antioxidant activity of chemical extract was $27.20 \pm 1.09 \mu \mathrm{mol}$ trolox $\mathrm{mL}^{-1}$, the same activity of physiological extract was decreased to 0.17 $\pm 0.02 \mu \mathrm{mol}$ trolox $\mathrm{mL}^{-1}$ in DPPH assay. The same values were consecutively $22.41 \pm 2.49$ and 3,09 $\pm 0.44 \mu \mathrm{mol}$ trolox mL $\mathrm{mL}^{-1}$ for FRAP assay; $21.09 \pm 1.65$ and $0.02 \pm 0.00 \mu \mathrm{mol}$ trolox $\mathrm{mL}^{-1}$ for CUPRAC assay. Ascorbic acid content of the herbal tea beverage was $28.39 \pm 1.84 \mathrm{mg} 100 \mathrm{~mL}^{-1}$. Beyond its nutritional and functional properties, Erica arborea herbal tea beverage is also preferred according to the panelists' sensorial evaluation.
\end{abstract}

\section{Phenolic Compound and Antioxidant Activity of Peruvian Propolis}

\author{
Eulalia Vargas Tapia* and Yong Kun Park \\ Department of Food Science, College of Food Engineering, State University of Campinas, Brazil
}

\begin{abstract}
The study was conducted to determinate the phenolic compounds and antioxidant activity of Peruvian propolis collected by Apis mellifera. The samples were analyzed as the phenolic contents showed significant differences at $5 \%$ of probability in the " $\mathrm{t}$ " student test. Mass spectrometry identify the presence of 3,5-diprenyl-4-hidroxycinnamic acid (Artepillin C). All samples presented high DPPH radical scavenging activity. This study demonstrated the great variety in chemical composition and biological properties of the Peruvian propolis.
\end{abstract}




\title{
Poster Presentations
}

\section{Biosynthesis of Red Cabbage Extract Directed Ag NPS and Their Effect on the Loss of Antioxidant Activity}

\author{
Ayse Demirbas ${ }^{1}$, Bruce A. Welt ${ }^{1}$ and Ismail Ocsoy ${ }^{2,3^{*}}$ \\ ${ }^{1}$ Department of Agricultural and Biological Engineering, University of Florida, FL, USA \\ ${ }^{2}$ Department of Analytical Chemistry, Faculty of Pharmacy, Erciyes University, Turkey \\ ${ }^{3}$ Nanotechnology Research Center, Erciyes University, Kayseri, Turkey
}

\begin{abstract}
Plants are sources of natural antioxidants that have been widely used as reducing and stabilizing agents for biosynthesis of nanoparticles (NPs). There are also many studies focused synthesizing nanoparticles via plants that is called "green synthesis" or "green chemistry". The biological synthesis of nanoparticles using plant extracts is desirable because of relatively inexpensive, environment-friendly, simple and natural means to minimize chemicals. Some studies suggest that plant extracts incorporated $\mathrm{NPs}$, such as Ag, enhanced antioxidant activity. In this study, we synthesized silver NPs (Ag NPs) with different concentrations of red cabbage (Brassica oleracea var. capitata) extract and measured how silver ions $\left(\mathrm{Ag}_{+}\right)$and $\mathrm{Ag} \mathrm{NPs}$ effect red cabbage antioxidant activity. We also characterized Ag NPs using UV-vis spectra, scanning electron microscopy (SEM) and Energydispersive X-ray spectroscopy (EDX). Results show that $\mathrm{Ag}_{+}$and $\mathrm{Ag}$ NPs extremely decrease antioxidant activity of anthocyanin towards 1,1-Diphenyl-2-picryl-hydrazyl (DPPH) antioxidant assay.
\end{abstract}

\section{Biotransformation of Limonene by the Fungi Aspergillus flavus, A. niger and Cunninghamella elegans}

\section{Iş1 Gazioğlu ${ }^{1,2^{*}}$, John B. Sutherland ${ }^{1}$ and Gonçalo Gamboa da Costa ${ }^{1}$}

${ }^{1} U$. S. Food and Drug Administration, National Center for Toxicological Research, Jefferson, AR, USA

${ }^{2}$ Bezmialem Vakif University, Faculty of Pharmacy, Department of Analytical Chemistry, Turkey

\begin{abstract}
Monoterpenes are important, abundant natural resources, which supply the solid raw materials for the flavor and food industries. Because of their significant bioactivities, some monoterpenes are used as drugs and have been found to have a wide range of beneficial effects on human health. D-Limonene ( $p$-mentha-1,8-diene) is a monocyclic monoterpene obtained from oranges, grapefruit and lemons. It exhibits a wide variety of pharmacological properties, including antioxidant, antiinflammatory and anticarcinogenic activities. In this study, biotransformation of $\mathrm{D}-(+)-$-limonene was investigated using three fungal cultures, Aspergillus flavus NRRL 500, Aspergillus niger NRRL 599 and Cunninghamella elegans ATCC 36112. The fungal metabolites of limonsene were analysed by HPLC-DAD, TLC and GC-MS. The major biotransformed products of $C$. elegans were trans- $p$-mentha-2,8-dienol and 4-isopropenyl-1-methyl-1,2-cyclohexanediol; the major product in cultures of $A$. flavus was kojic acid; and the major biotransformed product of $A$. niger was 4-isopropenyl-1-methyl-1,2-cyclohexanediol.
\end{abstract}

Also, cell-free homogenates of A. niger, A. flavus and C. elegans were prepared for determination of total protein concentration by the BCA (Bicinchoninic acid) protein assay. A. flavus had the highest protein content with $4.886 \mathrm{mg} / \mathrm{mL}$. This is the first study of the biotransformation of limonene by A. flavus, A. niger and C. elegans.

\section{Development of Navy Bean-Soybean Milk Substitute and Evaluation of Its Nutritional and Physical Properties}

\author{
Sean X. Liư, Mukti Singh, Ashley E. Wayman and James A. Kenar \\ United States Department of Agriculture, Agricultural Research Service, National Center for Agricultural Utilization Research, Peoria, \\ IL, USA
}




\begin{abstract}
Plant based milk substitutes have been gaining popularity with consumers. The industry is increasingly looking into the new sources of plant-based feedstock for making new healthful beverages. Pulses (dry beans and peas) are a class of underutilized food commodities that, like soybean, are part of legume family. Pulses contain many healthful and nutritionally important substances and consumption of pulses have long known to be associated with good cardiovascular health. In this study we explored the possibility of developing navy bean based milk substitutes. Recognizing the shortcomings of navy bean as feedstock for milk substitute, we examined the soybean-navy bean blends of 100:0, 80:20, 60:40, 20:80, and 0:100 ratios and processed using traditional kettle cooking and steam jet cooking. The physical properties (including beverage stability under different temperature settings) of these beverages were examined and their nutritional and anti-nutritional attributes were measured. It was found that from the point of view of development of a healthful and nutritious milk substitute using soybean-navy bean blends, the blends with $60 \%$ or less navy bean processed by steam jet cooking provide the best shelf stability in a refrigeration environment.
\end{abstract}

\title{
Optimization of Cranberry Juice Deacidification by Electrodialysis with Bipolar Membrane: Impact of Pulsed Electric Field Conditions
}

\author{
Stéphanie Pelletier, Élodie Serre, Sergey Mikhaylin and Laurent Bazinet
}

Université Laval, 2325 Rue de l'Université, Ville de Québec, Canada

\begin{abstract}
Cranberry is well recognized for its beneficial effects on human health, but the consumption of cranberry juice is limited due to its high acidity (high organic acid content) which is the cause of undesirable side effects such as diarrhea, vomiting and bloating. Therefore, acidity must be reduced to improve the product taste and reduce undesirable effects. The deacidification of juices by conventional electrodialysis and electrodialysis with bipolar membranes (EDBM) with direct current (DC), has shown to be very effective in comparison with chemical and conventional methods. Therefore, the objective of this project is to apply pulsed electric field (PEF) during EDBM to deacidify cranberry juice. The PEF procedure consists of applying an electric pulse and a pause consecutively for a given time, and presents a lot of advantages such as reducing clogging and increased current efficiency. Nine different pulse/pause combinations were tested: $10 \mathrm{~s} / 2 \mathrm{~s}, 1 \mathrm{~s} / 0.1 \mathrm{~s}, 10 \mathrm{~s} / 0.1 \mathrm{~s}, 6 \mathrm{~s} / 2 \mathrm{~s}, 10 \mathrm{~s} / 0.1 \mathrm{~s}, 2 \mathrm{~s} / 2 \mathrm{~s}$, $1 \mathrm{~s} / 1 \mathrm{~s}, 6 \mathrm{~s} / 0.1 \mathrm{~s}, 6 \mathrm{~s} / 1 \mathrm{~s}$. This approach of using PEFs with bipolar membranes has never been tested to date. According to our results, the deacidification of cranberry juice is about $20 \%$ faster with PEFs for $1 \mathrm{~s} / 1 \mathrm{~s}$ and $2 \mathrm{~s} / 2 \mathrm{~s}$ conditions in comparison with deacidification with DC. This faster decrease in deacidification would be due to a higher migration of citric acid in these two conditions. It's the first time, at our knowledge, that the efficiency of applying PEF for deacidification of juice is demonstrated. EDBM under PEF would be a green and sustainable avenue to fruit juice deacidification and preservation of their organoleptic characteristics.
\end{abstract}

\section{Investigation of the Biochemical Pathways of Antidiabetic Chromium(III) Supplements}

\author{
Kabir M. Uddin" and David J. Henry \\ Chemical and Metallurgical Engineering and Chemistry, Murdoch University, Murdoch, Australia
}

\begin{abstract}
Although, a number of scientific studies suggest that chromium(III) supplements are of minimal benefit to most people [1, 2], there is increasing evidence to suggest that $\operatorname{Cr}(\mathrm{III})$ complexes may have benefits as an insulin enhancing treatment in Type 2 diabetics. However, this has also raised concerns about the potential toxicity of these species. This study involves a mechanistic investigation of the substitution pathways of several chromiu(III) nutritional supplements (e.g. chromium chloride, chromium picolinate $(\mathrm{CrPic})$, and chromium( $\alpha$-amino acids) 3 using both experimental and state of the art computational chemistry techniques. The synthesized chromium(III) nutritional supplements were characterized by elemental analysis, AAS, ESI-MS, ATR-FTIR, Raman, UV-Vis, EPR, HPLC, and electro-chemistry.

Our initial computational investigations have focussed on the mechanistic pathways for aquation of chromium(III) chloride under acidic (stomach) and mild (duodenum and blood) conditions [3, 4]. Calculations reveal that aquation of $\mathrm{CrCl}_{3}$ is slow in an acidic environment and this limits speciation of the complex. However, at mild $\mathrm{pH}$, conjugate base complexes easily form leading to a significant number of species that may be involved in biochemical pathways. Aquation of $\mathrm{CrPic}$ supplement proceeds via a multi-step process that can also lead to a number of intermediates. These results provide a detailed understanding
\end{abstract}


of the mechanism for the hydrolysis of $\mathrm{Cr}$ (III) supplements, which could be useful in understanding the speciation of $\mathrm{Cr}$ (III) complexes, prior to binding to biomolecules in physiological environments.

\title{
References
}

1. Anderson AA, Polansky MM, Bryden NA, Canary JJ. 1991. Supplemental-chromium effects on glucose, insulin, glucagon, and urinary chromium losses in subjects consuming controlled low-chromium diets. Am J Clin Nutr 54(5): 909-916.s

2. Glinsmann WH, Mertz W. 1966. Effect of trivalent chromium on glucose tolerance. Metabolism 15(6): 510-520. doi: 10.1016/0026-0495(66)90111-9

3. Uddin KM, Ralph D, Henry DJ. 2015. Mechanistic investigation of halopentaaquachromium(III) complexes: Comparison of computational and experimental. Computational and Theoretical Chemistry 1070: 152-161.doi: 10.1016/j. comptc.2015.08.007

4. Uddin KM, Poirier A, Raymond DJ. 2016. Mechanistic study of the aquation of nutritional supplement chromium chloride and other chromium(III) dihalides. Computational and Theoretical Chemistry 1084: 88-97. doi: 10.1016/j. comptc.2016.03.016

\section{Evaluation of Bioactive Compounds and Dietary Fiber in Skin and Bagasse of Kiwi Fruit (Actinidia deliciosa)}

\author{
Sabrina Sauthier Monteiro ${ }^{*}$, Marcela Bromberger Soquetta ${ }^{2}$, Flávia Santi Stefanello ${ }^{2}$, Katira da Mota Huerta ${ }^{2}$, Claudia \\ Severo da Rosa ${ }^{2}$ and Nelcindo Nascimento Terra ${ }^{2}$
}

${ }^{1}$ Institute of Health and Biotechnology, Federal University of Amazonas, Brazil

${ }^{2}$ Federal University of Santa Maria (UFSM), Brazil

\begin{abstract}
The kiwi fruit originated in Asia and became popular worldwide due to its sensory and nutritional properties, such as a high level of fiber, minerals and antioxidant activity. The objective of this study was to analyze the physicochemical and microbiological properties, as well as the bioactive compounds, of flour made from the skin and bagasse of two varieties (Bruno and Monty) of kiwi fruit (Actinidia deliciosa) at two stages of maturation. To obtain flour, the skin and bagasse were dried in an oven at $35{ }^{\circ} \mathrm{C}( \pm 5)$, ground and sieved $(0,5 \mathrm{~mm})$. Four treatments were performed for the Bruno variety and four treatments were used for the Monty variety, which were as follows: FCV - green skin flour; FCM - ripe skin flour; FBV- green bagasse flour; and FBM - ripe bagasse flour. The flour made with kiwi fruit peel from both varieties showed higher levels of bioactive compounds and antioxidant activity that the flour made with bagasse from both varieties. The FCV from the Bruno variety had higher DPPH values and levels of phenolic compounds (1262.34 mg GAE/100 g flour), while the Monty variety showed higher FRAP values, vitamin C (189.06 mg/100 g flour), flavonoids (486.47 mg/100 g flour), chlorophylls (12.13 mg/100 g flour) and carotenoids ( $246.91 \mathrm{lg} / 100 \mathrm{~g}$ flour). Flour made from kiwi fruit skin can be used to reduce agro-industrial waste. This flour is a promising ingredient which can be used to enrich products providing dietary fiber and bioactive compounds, as well as antioxidant action.
\end{abstract}

\section{Sapota-Do-Solimões (Quararibea cordata): Physical Characterization of the Whole Fruit and Chemical Characterization of the Pulp}

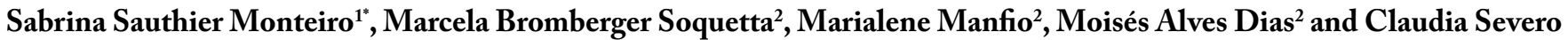 \\ da $\operatorname{Rosa}^{2}$
}

${ }^{1}$ Institute of Health and Biotechnology, Federal University of Amazonas, Brazil

${ }^{2}$ Federal University of Santa Maria (UFSM), Brazil

\section{Abstract}

The fruit of Amazon region sapota-do-Solimões is tasty and it is still little known, the fact that it has a thick peel has attracted interest for exportation. The aim of this study was to perform a biometric analysis of this fruit and also a chemical characterisation of the pulp of sapota-do-Solimões. The fruits were acquired in the Tefé region, AM, Brazil. They were harvested at a ripe stage and were transported to the Federal University of Santa Maria, RS, Brazil where they were selected, washed and 
sanitised. The percentage yields were calculated. The chemical composition and the mineral content of the pulp of the sapotado-Solimões were calculated. The percentages of peel, pulp and seeds were respectively 53.2\%, 39.6\% and 7.2\%. The transverse diameters of the fruits were positively correlated with the mass of the whole fruit, indicating that the greater the transverse diameter the greater would be the mass of the whole fruit. The chemical composition of $100 \mathrm{~g}$ of pulp of sapota-do-Solimões was as follows: $85.01 \%$ moisture; $0.79 \%$ ash; $0.67 \%$ protein; $0.10 \%$ lipids, $4.10 \%$ dietary fiber; $9.34 \%$ carbohydrate and 40.94 Kcal calories. The mineral magnesium $(23.69 \mathrm{mg}$ in $100 \mathrm{~g}$ of in natura sample) in the pulp showed the greatest relevance. The sapota-do-Solimões showed a high percentage of peel. The pulp is rich in dietary fiber and has a low caloric value. The level of magnesium in the pulp reached a good percentage (9\%) of the recommended daily intake.

\title{
Soybean Paste Development Enriched with Calcium and Fructooligosaccharides (FOS)
}

\author{
Luciana C. Nogueira*, Jessica B.M. Ramos and Patricia C.C. Averbug \\ Instituto Federal de Educação, Ciência e Tecnologia do Rio de Janeiro, Mestrado Profissional em Ciência e Tecnologia de Alimentos \\ (PCTA), Campus Rio de Janeiro, RJ, Brazil
}

\begin{abstract}
Despite the healthiness of appeal, the soy-based folders marketed in Rio de Janeiro have a low nutritional quality. This fact is due to the high starch content cassava and soybean oil used in their formulations. These raw materials are added to the formulation in order to reduce the cost of the product and mask the aftertaste of soybeans. Thus, the product would not meet the consumer looking for a soy-based food for the health benefits that it could provide. This study aims to develop a soybean paste enriched with calcium and Fructooligosaccharides, but adapted to the Western palate. The formulation of this product already has a differential, not only the presence of soy extract, but also soybeans, which impart a texture and nutritional and sensory characteristics differentiated, and not have added gluten. Calcium lactate were used and Raftilose at concentrations under Brazilian law. The product received additives for conservation under refrigeration and acidifying to $\mathrm{pH}$ correction. It was analyzed: physical chemical composition, storage and shelf life testing. Preliminary results point to changes in the interaction of $\mathrm{Ca}-\mathrm{FOS}$. The moisture content and protein percentage were the most affected parameters with the inclusion of FOS (+ 16\% and $-2 \%$, respectively); the calcium content significantly interfered with the ash content $( \pm 1 \%)$ and water activity (Aw) decreased by including $\mathrm{Ca}-\mathrm{FOS}(-0.01)$.
\end{abstract}

\section{Potential of Data Fusion and Chemometrics for Discrimination of Regional Provenance of Coffees Produced in Minas Gerais State, Brazil}

\author{
Adriana S. Franca ${ }^{1^{*}}$, Bruno Botelho $^{2}$ and Leandro S. Oliveira ${ }^{1}$ \\ ${ }^{1}$ DEMEC, Universidade Federal de Minas Gerais, Brazil \\ ${ }^{2}$ Centro Universitário de Belo Horizonte, Brazil
}

\begin{abstract}
Several attempts have been made to discriminate the coffee origin, given that its quality can vary considerably according to its geographic provenance. Although studies have been successful in discriminating coffees from distinct countries or distinct regions within a country, this is the first study dealing with the discrimination of coffees within a given region in a country. Minas Gerais State is the largest coffee producer in Brazil (50\% of the total country production), being divided into four coffee producing regions: Sul de Minas, Cerrado Mineiro, Matas de Minas and Norte de Minas. In this study, distinct techniques were evaluated for discrimination of green coffees from the aforementioned regions: FTIR, UV-Vis, fluorescence spectroscopy and mineral profile. Different extraction methods were tested (hot/cold water, microwave-assisted water extraction), with hot water being the most effective. Several organic solvents were tested (methanol, ethanol, ether, dichloromethane, acetone), with acetone standing out among them. The binary mixture acetone:water provided the best spectra for all the spectroscopic techniques employed. Discrimination models were developed based on the results obtained from the individual analytical techniques and also employing data fusion (merging of analytical information from the distinct techniques). The best discrimination results were obtained with UPLS-DA models based on fluorescence spectroscopy data, with high values of f-score (over 0.83 in the training sets). Data fusion did not improve model performance (f-scores ranged from 0.6 to 0.7 ) and thus was not able to eliminate the effect of poor discriminative data such as those based on mineral profile and UV-Vis.
\end{abstract}




\title{
Characterization of the Antioxidant Dietary Fiber Fraction of Coffee Production By- Products
}

\author{
Leandro S. Oliveira ${ }^{*}$, Lais Brito Cangussu ${ }^{2}$ and Adriana S. Franca ${ }^{1}$ \\ ${ }^{1} D E M E C$, Universidade Federal de Minas Gerais, Brazil \\ ${ }^{2} P P G C A$, Universidade Federal de Minas Gerais, Brazil
}

\begin{abstract}
Brazil is the leading producer and exporter of coffee in the world. Coffee processing generates significant amounts of byproducts, ranging from 30 to $50 \%$ in weight of total coffee production, with coffee husks and pulp being the major byproducts. A portion of these byproducts is currently being used in restricted applications, such as raw material for composting and combustion fodder for on-farm furnaces, and adequate disposal of the remainder portion constitutes a major environmental problem. However, coffee by-products are rich in carbohydrates, proteins and bioactive compounds, and thus should be envisioned as cheap renewable resources for a diversity of products. The aim of this study was to verify the feasibility of using coffee by-products derived from the coffee fruit, coffee pulp and husks, as sources of antioxidant dietary fibers, by chemically characterizing this fraction. Total dietary fiber content was similar to those of other fruit by-products (e.g., orange and lemon peels, and passion fruit seeds), being comprised predominantly of insoluble fiber ( 90\%). The amount of non-extractable phenolics ranged from 2 to 10 -fold the amount of extractable phenolics in the studied by-products, with the total amount of phenolics being significantly higher than those of other fruit by-products reported in the literature. The amount of non-extractable proanthocyanidins was about 2 to 4 times higher than that of the extractable ones. The observed antioxidant potential was similar to BHT, commonly employed as a standard in antioxidant potential evaluations. When compared to many residue-based fiber sources, coffee husks and pulp presented excellent technological properties.
\end{abstract}

\section{Chemical Indicators of Heat Treatment Applied to UHT Milk}

\author{
Paulo Henrique Fonseca da Silva ${ }^{*}$ and Leandra Natália de Oliveira Neves \\ Department of Nutrition, Federal University of Juiz de Fora, Brazil
}

\begin{abstract}
The chemical reactions that occur during milk heat treatment cause changes in pre-existing compounds and formation of new compounds, which can be used as indicators of the thermal process. The monitoring of these indicators in UHT milk can suggest technological interventions in order to reduce production costs and nutritional losses. This study aimed to quantify whey protein nitrogen index (WPNI) and 5-hydroxymethylfurfural (HMF) in UHT milk samples, with regular (n = 18) and reduced-lactose content $(n=3)$, sold in the Brazilian market. Analytical methodologies for WPNI and HMF (both measured by spectrophotometric methods) were implemented. The adjustment of mathematical models regarding the calibration curves were considered statistically appropriate. The mean value for WPNI were $2.32 \pm 1.41 \mathrm{mg} \mathrm{WPN} . \mathrm{mL}^{-1}$. The mean values for free HMF were $2.83 \pm 0.44 \mu \mathrm{mol} . \mathrm{L}^{-1}$ and $6.45 \pm 0.44 \mu \mathrm{mol} . \mathrm{L}^{-1}$ for regular and reduced-lactose milk, respectively. The mean values for total HMF were $12.03 \pm 0.36 \mu \mathrm{mol} . \mathrm{L}^{-1}$ and $137.75 \pm 5.47 \mu \mathrm{mol} . \mathrm{L}^{-1}$ for regular and reduced-lactose milk, respectively. The analysis of variance for all parameters showed differences statistically significant. The WPNI indicated that $76 \%$ and $24 \%$ of the samples are classified as medium and high heat treatment, respectively. The HMF contents were able to discriminate samples with regular and reduced-lactose. Fluctuations in WPNI and HMF contents observed suggested the occurrence of non-uniformity of the UHT processing. The analytical methods used are able to evaluate the thermal process and allowing studies about the chemical characteristics of the samples.
\end{abstract}

\section{Measurement of Calcium Partition in "Minas Padrão" Soft Cheese by Flame Atomic Absorption Spectrometry and Ion-Selective Electrode Potentiometry}

\author{
Paulo Henrique Fonseca da Silva* and João Pablo Fortes Pereira \\ Department of Nutrition, Federal University of Juiz de Fora, Brazil
}

\section{Abstract}

"Minas Padrão" (MP) cheese is a typical Brazilian product. Calcium in cheese is found complexed with proteins or soluble 
in aqueous phase as a free form or associated with anions. This study aimed to evaluate the calcium partition in MP soft cheeses marketed in Juiz de Fora - Brazil. It was performed compositional analysis, determination of total calcium, calcium in the aqueous phase, free calcium and $\mathrm{pH}$ in cheeses with 28 days after manufacture. The moisture was determinated by gravimetric analysis, fat content by Gerber's method and fat in dry matter by ratio between fat and dry matter. The total calcium and calcium in the aqueous phase were determinated by Flame Atomic Absorption Spectrometry and the aqueous phase extraction was carried out by mechanical pressing of cheese samples. The determination of free calcium was performed in the aqueous phase by ion-selective electrode potentiometry. The $\mathrm{pH}$ of cheeses was performed by potentiometry. Both (ion-selective and $\mathrm{pH}$ equipment) were properly calibrated with standard solutions. The mean values found were: moisture 32.4 $\pm 3.8 \%$; protein $22.5 \pm 1.4 \%$; fat $26.7 \pm 1.9 \%$; fat in dry matter $46.3 \pm 2.7 \%$; total calcium $687 \pm 86 \mathrm{mg} .100 \mathrm{~g} \mathrm{~g}^{-1}$; calcium in aqueous phase $482 \pm$ $139 \mathrm{mg} .100 \mathrm{~g}^{-1}$; free calcium $99.3 \pm 10.7 \mathrm{mg} .100 \mathrm{~mL}^{-1}$; pH $5.14 \pm 0.20$. Approximately $70 \%$ of the calcium content in cheeses is in the aqueous phase, associated with salts. This is an important characteristic concerning techno-functional and nutritional properties of MP cheese. The $\mathrm{pH}$ value is important in calcium partition, since that the decrease of $\mathrm{pH}$ results in increase of calcium content in aqueous phase.

\title{
Effect of Processing on the Nutritional Properties of Ibyer-I-Angen: a Traditional Cereal Gruel Made from Pearl Millet (Pennisetum glaucum)
}

\author{
Bridget Dooshima Igbetar ${ }^{*}$, Christine Bosch and Caroline Orfila \\ School of Food Science and Nutrition, University of Leeds, England, UK
}

\section{Abstract}

Ibyer-I-Angen is an indigenous, generally fermented gruel made from pearl millet and commonly consumed by the Tiv ethnic group in Benue State, Nigeria. Pearl millet is commonly consumed as a staple cereal in parts of Asia and Africa. It is a drought resilient crop that has been reported to be the fourth most produced crop in the world, with India, Niger, Nigeria and Mali being some of the largest producers. There has been no published data and information on the processing or physiological behaviour of Ibyer-I-Angen. Consequently, this research attempts to explore the effect of flour particle sizes and the impact of processing on in vitro starch digestibility of Ibyer-I-Angen. The experimental hypotheses are particle sizes and processing of Ibyer-I-Angen will result in delayed starch digestion and slower glucose absorption.

Flour of various particles sizes (between 250-1000 microns) were made by milling and sieving of pearl millet grains. Porridge was made from these flours and the moisture content, soluble sugar content, starch content and in vitro starch digestibility were measured. The in vitro starch digestibility was assessed by analysis of glucose released during digestion with $\alpha$-amylase and amyloglucosidase by high performance anion exchange chromatography with pulsed amperometric detection (HPAEC-PAD). Glucose, fructose, sucrose and maltose were detected as free sugars present in millet porridge. The levels of free sugar were very low, below $1 \%$.

\section{Development of Novel Earthworm Dietary Supplements Using Pressure Treatment}

\author{
Shin-ichi Akazawa ${ }^{1 *}$, Shimizu Mika1 and Shinnosuke Wakimoto \\ ${ }^{1}$ National Institute of Technology, Nagaoka College, 888 Nishikatakaimachi, Nagaoka, Niigata Prefecture, Japan \\ ${ }^{2}$ Waki Pharmaceutical Co., Ltd., Japan
}

\begin{abstract}
Earthworms are well-known soil decomposers. They have various digestive enzymes because they are polyphagous animals. Earthworms have also been studied for their therapeutic effects against human diseases. Especially, earthworms have potent fibrinolytic enzymes called as Lumbrokinase. The enzyme is very stable even if leave it out at room temperature, and also dried earthworm powder has been found fibrinolytic activity. Therefore, we have developed a novel earthworm dietary supplement having fibrinolytic activities using high-pressure treatment. However, some digestive enzyme activities were lost by high-pressure treatment. Since digestive enzymes such as amylase are also useful for our health, we investigate these enzymes activities under the low-pressure treatment. The earthworms, Eisenia fetida Waki, were starved for $24 \mathrm{~h}$. The starved earthworms were washed and disrupted by a mixer. The mixture was centrifuged. After that, pressure and heat treatments against the supernatant were performed at various hours. The treated sample was centrifuged, and the supernatant was used for various enzyme assay. As a result, low-pressure treatment did not have an enhancing effect of earthworm endoglucanase, which is one of the cellulases, activity. On the other hand, amylase and lumbrokinase activities were enhanced by low-pressure treatment. These results show that the low-pressure treatment may be useful as a development of earthworm dietary supplement.
\end{abstract}




\title{
Differential Effects of Vanillin and Syringaldehydde from Seed of Japanese Apricot on 3T3-L1 Adipogenesis
}

\author{
Yoshiharu Okuno $^{1}$, Saki Nomura ${ }^{1}$, Shohei Yamato ${ }^{1}$, Ryohei Kono ${ }^{2}$, Sachiko Nomura ${ }^{2}$, Akihiro Maeno ${ }^{2}$ and Hirotoshi \\ Utsunomiya $^{2}$
}

${ }^{1}$ National Institute of Technology Wakayama College, Japan

${ }^{2}$ Wakayama Medical University, Japan

\begin{abstract}
The Japanese apricot (Prunus mume Sibe. et. Zucc.; Ume in Japanese), a deciduous tree of the family Rosacea, originated in the central and southern regions of China, and has now 400-500 varieties worldwide. The fruits of Japanese apricot are taken in foods as Umeboshi, Bainiku-ekisu, Ume liquor, ume-based soft drinks, and so on. The fruit has been known to have various biological activities, and the fruit has been prescribed medicine for disorder of the stomach and intestines, quick recovery from fatigue, cough and diarrhea in Chinese traditional prescriptions. However, very few reports are available that proofs of components from Japanese apricot are effective against diseases. During the screening program to discover such compounds from natural products, a dichloromethane extract from seed of Japanese apricot enhanced the differentiation of 3T3-L1 preadipocyte. In this study, we report the isolation and identification of differentiation-promoting compounds against 3T3-L1 preadipocyte from seed of Japanese apricot. Dichloromethane extract was fractionated by $\mathrm{SiO}_{2}$ column chromatography using differentiation assay as a guide. Active compounds 1 and 2 were isolated and identified as vanillin (1) and syringaldehydde (2) by HRESI-MS and ${ }_{1} \mathrm{H}$ NMR spectroscopy. Effect of enhanced differentiation of compounds 1 and 2 increased each about 2 and 3 fold that of negative control at concentration of $1 \mathrm{mM}$. In addition, compound 2 induced GPDH activity in 3T3-L1 cells.
\end{abstract}

\section{Apoptosis-Including Components from Pickled Prunus mume Sieb. Et Zucc}

\author{
Honoka Wada $^{1^{*}}$, Yoshiharu Okuno ${ }^{1}$, Ryouhei Kono ${ }^{2}$, Sachiko Nomura ${ }^{2}$, Akihiro Maeno ${ }^{2}$ and Hirotoshi Uthunomiya ${ }^{2}$ \\ ${ }^{1}$ National Institute of Technology, Wakayama College, Japan \\ ${ }^{2}$ Wakayama Medical University, Japan
}

\begin{abstract}
Apoptosis inducing components on human stomach cancer MKN45 from pickled Prunus mume SIBE et. Zucc (Umeboshi in Japanese) was investigated. Isolation of the active compounds was carried out MTS assay on MKN45 as guide. Hexane extract and dichloromethane extract of Umeboshi showed anti-proliferative activity on MKN45, and these extracts were fractionated by $\mathrm{SiO}_{2}$ column chromatography. Fr. $\mathrm{H} 3$ from hexane extract showed anti-proliferative activity, but it couldn't be isolated active compound. On the other hand, active compound 1 was isolated and identified as dimethyl malate (1) by 1H, 13C NMR and GC-MS spectroscopy from dichloromethane extract. Compound 1 showed anti-proliferative activity at concentration-dependent and cytotoxic effects above at concentration of $310 \mu \mathrm{M}$ in MKN45 cells. Therefore, effects of compound 1 against the mitochondrial transmembrane potential, induction of caspase 3/7 and chromatin condensation effect were demonstrated. Compound 1 showed disappearance of the mitochondrial transmembrane potential at concentrationdependent manner. Caspase 3/7 was induced 1.5 to 3.0 times the control by compound 1 . Furthermore, compound 1 induced chromatin condensation. From these results, Compound 1 induced apoptosis in MKN45, and its mode of action is activation of the caspase $3 / 7$ through a mitochondrial pathway.
\end{abstract}

\section{Antiobesity Compounds from Citrus jabara}

\author{
Shiho Ohta* and Yoshiharu Okuno \\ National Institute of Technology, Wakayama College, Japan
}

\section{Abstract}

Citrus jabara is a type of sour citrus fruit and indigenous to Kitayama-village, Wakayama, Japan. C. jabara has the effect against cedar pollinosis. However, other biological activities of C. jabara have been not reported. Therefore, inhibitory effects of C. jabara peel water extract (JWE) on lipid accumulation of 3T3-L1 adipocytes were investigated.

3,5,6,7,8,3',4'-Heptametoxyflavone (1), 3-hydroxy-5,6,7,8,3',4'-hexametoxyflavone (2) and 3,5,6,7,3',4'-hexametoxyflavone 
(3) were isolated from JWE as a guide of lipid accumulation on 3T3-L1 adipocytes. The effects of Compounds 1-3 on cellular lipid accumulation in 3T3-L1 adipocytes was determined using Oil red $\mathrm{O}$ staining. Lipid accumulation of 3T3-L1 adipocytes treated with $200 \mu \mathrm{M}$ of compounds 1-3 was decreased about 50, 50, $10 \%$ to untreated adipocytes, respectively. To determine the antiadipocytic effect of compounds 1-3 on 3T3-L1 adipocytes, we also measured glycerol-3-phosphate dehydrogenase (GPDH) activity. Compounds 1-3 were shown to suppress the GPDH activity in a dose-dependent manner. Especially, the GPDH activity when compound $3(200 \mu \mathrm{M})$ was added to cells significantly lower than the GPDH activity in untreated cells. On this results, it is indicated that compounds 1-3 inhibited lipid accumulation by blacking GPDH activity in adipocytes. Moreover, lipolytic activity of compounds 1-3 on mature 3T3-L1 adipocytes was determined by measuring glycerol levels that released in the medium. Compounds 1-3 treatment increased glycerol secretion in a dose-dependent manner. Especially, compound $1(200 \mu \mathrm{M})$ strongly stimulated lipolysis in 3T3-L1 adipocytes.

\title{
Evaluation of B-Carotene Contents of Selected Blanched Vegetables Commonly Consumed in South Korea
}

\author{
Jung-Ah Shin ${ }^{1^{*}}$, Hyeon-Hwa Lee ${ }^{1}$, Youngmin Choi ${ }^{2}$ and Ki-Teak Lee ${ }^{1}$ \\ ${ }^{1}$ Department of Food Science and Technology, Chungnam National University, Daejeon, South Korea \\ ${ }^{2}$ Functional Food E'Nutrition, Dept. Agro-Food Resource, NationalAcademy of Agricultural Science, RuralDevelopment Administration, \\ Jeonju, South Korea
}

\begin{abstract}
In this study, $\beta$-carotene content databases of 14 kinds of vegetables commonly consumed in South Korea were constructed on raw and blanched vegetables. The content and true retention factor of $\beta$-carotene on leaf and stalk vegetables, paprika, sweet pepper and tomato after blanching were investigated and compared. Certified reference material (CRM, mixed vegetables BCR-485) was used to validate the accuracy and precision of the HPLC method used (RSD of 2.5\% and a recovery percentage of 99.6\%). True retention factor and percent variation of $\beta$-carotene content after blanching were obtained and compared. The blanching increased generally $\beta$-carotene contents in leaf vegetables probably due to the release of bound $\beta$-carotene and the minimal effect of a short cooking time $(30 \mathrm{sec})$ on $\beta$-carotene stability. True retention factors of leaf vegetables were ranged from 119.3 to $238.2 \%$. After blanching, the weights of stalk vegetables (Allium hookeri, Chamjukramul, sweet potato stalks with or without skin, and garlic flower stalk, except for Sebalramul) decreased a little while $\beta$-carotene contents noticeably increased with ranges from 34.7 to $176.4 \%$. Comparatively, high correlation between $\beta$-carotene content and true retention factor in blanched stalk vegetables was observed. In other words, true retention factor decreased when the content of $\beta$-carotene increased in stalk vegetables after blanching. This paper can give an account of the changes of $\beta$-carotene contents by blanching in various vegetables for providing the extensive information on nutritional value of $\beta$-carotene content.
\end{abstract}

\section{Analysis of Biogenic Amines and Biogenic Amine-Producing Bacteria in Baechukimchi and Kkakdugi, Korean Traditional Fermented Vegetable Products}

\author{
Bo Young Byun and Jae-Hyung Mah* \\ Korea University, 145 Anam-ro, Seongbuk-gu, Seoul, South Korea
}

\begin{abstract}
Ten Baechukimchi (cabbage kimchi) and ten Kkakdugi (diced radish kimchi) products were analyzed for the contents of biogenic amines (tryptamine, $\beta$-phenylethylamine, putrescine, cadaverine, histamine and tyramine) and tested to determine microbial characteristics. Baechukimchi and Kkakdugi products had relatively higher levels of histamine $(9.54 \pm 6.77$ and 8.97 $\pm 7.29 \mathrm{mg} / \mathrm{kg}$, respectively) and followed by tyramine $(3.10 \pm 2.19$ and $4.13 \pm 4.51 \mathrm{mg} / \mathrm{kg}$, respectively), while other biogenic amines were usually much less than $5.0 \mathrm{mg} / \mathrm{kg}$. The lactic acid bacterial counts of Baechukimchi and Kkakdugi products ranged from $10^{5} \mathrm{CFU} / g$ to $10^{6} \mathrm{CFU} / g$, respectively. Meanwhile, a total of 40 bacterial strains were isolated from Baechukimchi and Kkakdugi products which had been manufactured by medium-sized firms in Korea and analyzed for their abilities to produce biogenic amines in an assay medium. The cultures of these strains contained relatively higher levels of histamine and tyramine, while other biogenic amines were mostly less than $1.0 \mu \mathrm{g} / \mathrm{ml}$. The strains isolated from Baechukimchi were identified to be Leuconostoc mesenteroides (65\%), Lactobacillus sakei (25\%), L. plantarum (5\%) and L. salivarius (5\%), while the strains isolated from Kkakdugi were all identified to be L. mesenteroides. In conclusion, the amounts of biogenic amines in Baechukimchi and Kkakdugi appeared to be within safe levels for consumption. This study further indicates that lactic acid bacteria like Lactobacillus spp. and Leuconostoc spp. are the primary contributors to both histamine and tyramine contents in kimchi and that, as bacterial diversity is lower in the food, their activity to produce biogenic amines is similar.
\end{abstract}




\title{
Analysis of Biogenic Amines and Biogenic Amine-Producing Bacteria in Gochujang and Cheonggukjang, Korean Traditional Fermented Soybean Pastes
}

\author{
Bo Young Byun and Jae-Hyung Mah* \\ Korea University, 145 Anam-ro, Seongbuk-gu, Seoul, South Korea
}

\begin{abstract}
Ten Gochujang and ten Cheonggukjang products were analyzed for the contents of biogenic amines (tryptamine, $\beta$-phenylethylamine, putrescine, cadaverine, histamine and tyramine) using HPLC. The biogenic amine contents were significantly higher in Cheonggukjang products than in Gochujang products. Cheonggukjang products had especially high levels of vasoactive monoamines including tryptamine $(56.74 \pm 74.25 \mathrm{mg} / \mathrm{kg}), \beta$-phenylethylamine $(155.63 \pm 221.81 \mathrm{mg} /$ $\mathrm{kg}$ ) and tyramine $(108.41 \pm 92.58 \mathrm{mg} / \mathrm{kg})$. It is noteworthy that, regarding the toxicity of these monoamines, $30 \mathrm{mg} / \mathrm{kg} \mathrm{of}$ $\beta$-phenylethylamine and $100-800 \mathrm{mg} / \mathrm{kg}$ of tyramine have been reported to be toxic doses in foods, respectively. The viable cell counts of Gochujang and Cheonggukjang products were $10^{6}$ and $10^{9} \mathrm{CFU} / \mathrm{g}$, respectively. Meanwhile, a total of 40 bacterial strains were isolated from Gochujang and Cheonggukjang products which had been manufactured by medium-sized firms in Korea and analyzed for their abilities to produce biogenic amines using HPLC. The cultures of these strains contained significantly higher levels of tyramine, while other biogenic amines were usually less than $1.0 \mu \mathrm{g} / \mathrm{ml}$. The strains isolated from Gochujang were all identified to be Bacillus subtilis, while the strains isolated from Cheonggukjang were identified to be B. subtilis (35\%), B. licheniformis (30\%) and B. coagulans (5\%). Taken together, the contents of biogenic amines in Gochujang and Cheonggukjang are predominantly due to the strains capable of producing biogenic amines, and Bacillus spp. seem to be contributors to tyramine contents in these foods. The results further indicate that bacterial activity to produce biogenic amines is more similar, as their diversity is lower in food.
\end{abstract}

\section{Quality Characteristics of Low-Malt Beer Produced by the Addition of Sweet Potato Paste}

\author{
Dong-Seong Choi ${ }^{12^{*}}$,Jeong-Seob Park ${ }^{3}$, Eun-Bi Oh${ }^{2}$ and $\mathrm{Ha}-\mathrm{Na} \mathrm{Yang}{ }^{4}$ \\ ${ }^{1}$ Department of Food E Biotechnology, Woosuk University, Korea \\ ${ }^{2}$ Department of Food Science E' Biotechnology, Graduate School, Woosuk University, Korea \\ ${ }^{3}$ Daedoo Foods Co., LTD, Korea \\ ${ }^{4}$ GDO Cake Co., LTD, Korea
}

\begin{abstract}
Sweet potato-malt worts were prepared by using malt (a main ingredient), sweet potato paste of Shinyulmi and Shinjami (a main adjunct), enzymes, hop, and water. And we brewed low malt-beers of lager or ale type by using these worts and inoculating bottom and top fermenting yeasts, respectively. Moreover the componential and functional characteristics of the resulting beers were evaluated. During saccharifying the paste of sweet potato, an enzyme agent containing $\alpha$-amylase was added to improve the filterability and to increase of total sugar. The sugar content of sweet potato-malt wort that prepared by the addition of $0.1 \%$ enzyme agent containing $\alpha$-amylase and three step infusion procedure was $13.5^{\circ} \mathrm{Brix}$ which was an adequate sugar level for beer brewing. In all of lager- and ale-type low-malt beers using $41.6 \%$ of sweet potato pastes, the sensory attributes very similar to that of $100 \%$ malt beer were obtained and were very good that had unique sweetness, mellowness, and color. The polyphenol and anthocyanin contents in Shinjami beer were increased with increasing content of the paste, also increased DPPH and ABTS radical scavenging activities. But in Shinyulmi beer, the polyphenol and anthocyanin contents were decreased with increasing content of the sweetpotato paste. A strong correlation between antioxidant activities and polyphenol and anthocyanin contents in sweet potato beers had been observed.
\end{abstract}

\section{Antimicrobial Activity and Deodorization Effect of the Extracts of Mulberry Leaves}

\author{
Eun-Bi Oh ${ }^{1^{*}}$ and Dong-Seong Choi ${ }^{1,2}$ \\ ${ }^{1}$ Department of Food Science E' Biotechnology, Graduate School, Woosuk University, Korea \\ ${ }^{2}$ Department of Food E' Biotechnology, Woosuk University, Korea
}




\begin{abstract}
Mulberry leaves contain polyphenols and have various biological active compounds. In this study, we examined the antibacterial activity and deodorization effect of water and $80 \%$ ethanol extracts of the leaves of two different mullberry species (Gwasang No. 2 and Cheongil). The antibacterial activities were tested against Shewanella putrefaciens KCTC 12458, Proteus mirabilis KCTC 2566 and Vibrio fuvialis KCTC 2473 using paper disk and agar dilution methods. While, the deodorizing effects were evaluated against the distinctive odor of ammonia and trimethylamine of shellfish using gas detector tubes. The mulberry leaves extracts demonstrated antibacterial activities against all the strains of bacteria except for the $80 \%$ ethanol extracts, which had no effect against $P$. mirabilis. The minimal inhibitory concentration (MIC) values were found to be in the range of $10-200 \mathrm{mg} / \mathrm{mL}$. The mulberry leaves extracts exhibited the $63-76 \%$ and $46-62 \%$ decrease in the amounts of ammonia and trimethylamine, respectively, showing the effective deodorizing activity of that mulberry leaves extracts.
\end{abstract}

\title{
Quantitative Analysis of 2- and 4-Methylimidazole in Cola and Dark Beer by Gas Chromatography-Triple Quadrupole Tandem Mass Spectrometry
}

\author{
Sol Ji Choi", Hee Jeong Park and Mun Yhung Jung \\ Department of Food and Biotechnology, College of Food Science, Woosuk University, Samnye-eup, Wanju-gun, Jeonbuk Province, \\ Republic of Korea
}

\begin{abstract}
A fast quantitative analytical method for the analysis of 2-methylimidazole (2-MeI) and 4-methylimidazole (4-MeI) in colas and dark beers by a gas chromatography-triple quadrupole tandem mass spectrometry (GC-MS/MS) following derivatization with isobutylchloroformate (IBCF) has been developed. This technique does not require sample concentration, the ion pair extraction, and back-extraction steps. This method provided high linearity, low LOD and LOQ, high recovery, and high intra- and inter-day repeatability. Internal standard method with diluted stable isotope (4-MeI-d6) and 2-ethylimidazole (2-EI) could not correctly compensate the matrix effects. Standard addition technique was found to be appropriate for the quantification of 2- and 4-MeI. The established method was successfully applied to colas and dark beers for the determination of 2-MeI and 4-MeI. The 4-MeI contents in colas and dark beers ranged from 8 to $319 \mathrm{mg} / \mathrm{L}$ and from trace to $417 \mathrm{mg} / \mathrm{L}$, respectively. Small quantity $(0-8 \mathrm{mg} / \mathrm{L})$ of $2-\mathrm{MeI}$ was found only in dark beers. The contents of $4-\mathrm{MeI}(22 \mathrm{mg} / \mathrm{L})$ in colas obtained from fast food restaurants were significantly lower than those $(177 \mathrm{mg} / \mathrm{L})$ in canned or bottled colas.
\end{abstract}

\section{Characterization and Quantification of Isoflavones in Soybean Products by High Performance Liquid Chromatography-Electrospray Ionization-Quadrupole-Time of Flight-Mass Spectrometry (HPLC-ESI-QTOF-MS)}

\author{
Ju Hee Kang;, Mi Jin Lee and Mun YhungJung \\ Department of Food and Biotechnology, College of Food Science, Woosuk University, Samnye-eup, Wanju-gun, Jeonbuk Province, \\ Republic of Korea
}

\begin{abstract}
A HPLC-ESI-QTOF-MS analytical method was established for the characterization and quantification of 12 isoflavones in soybean products. Identification of the isoflavones were based on the accurate mass data of their respective protonated mass ions, $\mathrm{Na}$ or $\mathrm{K}$ adduct ions, and fragment ions. Isotope ion patterns of the mass spectra were used for the confirmation of the identities. Extracted ions of the protonated mass ions of the isoflavones were used for their quantification. It took only $12 \mathrm{~min}$ to separate and quantitate the twelve different isoflavones with the present method. The established LC-QTOF/MS was an effective analytical method for the simultaneous characterization and quantification of isoflavones with short analytical time, high selectivity, a high linearity $\left(\mathrm{r}^{2}>0.992\right)$, low LOD and LOQ, high precision, inter-and intra-day repeatability, and no significant matrix effect. The method requires simple sample preparation procedure (solvent extraction, dilution, and syringe filtration). The method was successfully applied to measure the quantities of 12 isoflavones in various soybean products (various beans, tofu, and soymilks).
\end{abstract}




\title{
Comparative Protective Effect of Gallic Acid on the Oxidation of Vegetable Oils during High Temperature Heating with Synthetic Antioxidants
}

\author{
Min Kyung Lee, Mun Yhung Jung and Dong Seong Choi \\ Department of Food and Biotechnology, Graduate School, College of Food Science, Woosuk University, Wanju-Gun, Jeonbuk, Republic \\ of Korea
}

\begin{abstract}
The comparative protection activity of gallic acid with well-known synthetic antioxidants (tert-butylhydroquinone, butylated hydroxylanisole, propylgallate) on the oxidations of vegetable oils during heating for $8 \mathrm{~h}$ at $180{ }^{\circ} \mathrm{C}$ were studied. Gas chromatographic analysis of fatty acid composition, spectrophotometric analysis of conjugated dienoic acid contents, and high performance size exclusion chromatographic analysis of polymers in the oils were performed for monitoring the degree of oxidation of the oils. The quantitative effects of gallic acid at three different concentrations on the time-course thermal oxidations in the two different vegetable oils were also studied for 6 days at $180^{\circ} \mathrm{C}$. Gallic acid at $200 \mathrm{ppm}$ showed higher protective activity than the synthetic antioxidants at the same concentration. Gallic acid also exhibited persistently strong protective activity, in a concentration dependent manner, on the changes in oxidation indices in corn and soybean oils throughout the prolonged heating period of 6 days at $180{ }^{\circ} \mathrm{C}$. The present results clearly suggested that gallic acid would be a potential natural substitute of synthetic antioxidants for the protection of vegetable oils during high temperature heating.
\end{abstract}

\section{Ultra-High Performance Liquid Chromatography-Tandem Mass Spectrometry in Multiple Reaction Monitoring Mode for the Multi-Residue Pesticide Analysis in Rice}

\author{
Je Young Shin", Hee Jeong Park and Mun Yhung Jung \\ Department of Food and Biotechnology, Graduate School, Woosuk University, Jeonbuk, Republic of Korea
}

\begin{abstract}
A fast ultra-high performance liquid chromatography-tandem mass spectrometry (UHPLC-MS/MS) in multiple reaction monitoring (MRM) mode was successfully established for the multi residue pesticide analysis (103 pesticides) in agricultural products and foods. A reverse phase column was used for the separation of pesticides. Pesticides in rice were extracted and purified by QuEChERS extraction/purification method. Full separation and identification of the 103 pesticides were achieved within 3 min of UHPLC-MS/MS running time. The single mass spectrometry with full scan mode and tandem mass spectrometry with product ion scan mode were conducted to select the transitions for the optimum quantification sensitivity of pesticides. This established analytical method showed the very low limit of detection (LOD) for the pesticides. The calibration curves obtained by this method showed excellent linearity $\left(r^{2}=0.993-0.999\right)$ of most of the tested pesticides with a few exception $\left(r^{2}=0.984\right)$ in an established concentration range. High recovery rates with the known amount spiked samples were obtained. This method could be successfully applied to analyze the multi residue pesticides in rice samples.
\end{abstract}

\section{Effects of Methanolic Extract of Clove and Its Fraction on the Thermal Oxidation of Oils at $180^{\circ} \mathrm{C}$}

\author{
Ji Su Park ${ }^{1,2^{*}}$ and Mun Yhung Jung ${ }^{1}$ \\ ${ }^{1}$ Department of Food and Biotechnology, Graduate School, Woosuk University, Wanju-Gun, Jeonbuk, Republic of Korea \\ ${ }^{2}$ Food Analysis Center, Korea Food Research Institute, Seongnam-si, Kyonggi-do, Republic of Korea
}

\begin{abstract}
Vegetable oils are frequently used as cooking media for deep-fat frying. High temperature heating induces fast thermal oxidation, resulting in the loss of unsaturated fatty acids and degradation of color, flavor, and nutritional quality of oil and formation of toxic components. The effects of clove methanol extracts on the thermal oxidation of vegetable oils during 8 days heating at $180{ }^{\circ} \mathrm{C}$ were studied by analyzing the changes of fatty acid composition, conjugated diene contents, and polymer formation in vegetable oils. Clove extract exhibited strong inhibitory activity on the compositional changes of fatty acids and formations of conjugated dienoic acid and polymers in the vegetable oils. The $1.0 \%$ clove methanol extract exhibited significantly
\end{abstract}


higher protective activity than that of $0.02 \%$ TBHQ on the changes of oxidation indices in vegetable oils during heating at $180{ }^{\circ} \mathrm{C}$. The crude extract of clove was successively fractionated into diethyl ether, ethyl acetate and butanol fractions. Ethyl acetate fraction had the highest antioxidative effects on thermal oxidation of vegetable oils. The results suggested that methanolic extract of clove and its ethyl acetate fraction may be used for the protection of high temperature heat induced oxidation of vegetable oils.

\title{
Antioxidant Properties of Fermented Cactus Plant (Opuntia humifusa) with Fruit Peel Waste
}

\author{
So Jin Yong ${ }^{*}$ and Jiyeon Chun \\ Department of Food Science and Technology, Sunchon National University, South Korea
}

\begin{abstract}
Opuntia humifusa (genus Opuntia) called prickly pear cactus is known to possess various nutraceutical properties. This plant is continuously being studied in order to maximize its utilization. In addition to a plant's fruit, many fruit peels have been known to contain various functional components. Therefore, in this study, O. bumifusa (Oh) was fermented with pomegranate (Punica granatum L.) rind and yuzu (Citrus junos) peel (P and $\mathrm{Y}$, respectively) at $37{ }^{\circ} \mathrm{C}$ for about 7 days, and their physicochemical and antioxidant properties were investigated. Mixing ratios for fermentation were Oh:P (or Y):sucrose = 9:1:10 (w/w/w) for $\mathrm{Oh}-\mathrm{P}$ and $\mathrm{Oh}-\mathrm{Y}$ (Oh:sucrose=1:1 for $\mathrm{Oh}$, control). Increase in viable count of lactic acid bacteria was observed as fermentation progressed; $\mathrm{pH}$ slightly decreased due to acid production as a slight decrease in sugar content was observed, from about 20 to $15^{\circ}$ Brix. Oh-P showed the highest DPPH radical scavenging activity after $7 \mathrm{~d}$-fermentation $(97.24 \mu \mathrm{g}$ GAE/mL), which was high seven folds compared to fermented $\mathrm{Oh}$ (control, $<1 \mu \mathrm{g}$ GAE/mL). Reducing power of Oh-P $(117.51 \mu \mathrm{g}$ GAE/mL) was also higher than the other fermented samples by about five folds. Total phenolic content in Oh-P was also the highest (159.29 $\mu \mathrm{g} \mathrm{GAE} / \mathrm{mL}$ ) among the treatments. In conclusion, pomegranate rind is a potential source of functional compounds which can be used in the production of fermented cactus plant with increased beneficial antioxidant components.
\end{abstract}

\section{Evaluation of Phytosterol and Vitamin Contents in Opuntia bumifusa grown in South Korea}

\author{
So-Hyeong $\mathrm{Oh}^{*}$ and Jiyeon Chun \\ Department of Food Science and Technology, Sunchon National University, South Korea
}

\begin{abstract}
Opuntia humifusa is a type of cactus plant (family Cactaceae, genus Opuntia). It is known to contain health benefits and fight against diseases like diabetes mellitus and hypertension. In order to obtain basic information on beneficial components of $\mathrm{O}$. humifusa grown in South Korea, general composition, phytosterols and vitamins of O. humifusa stem and fruit were analyzed. O. humifusa stem showed higher moisture, ash and fat contents but its fruit had higher protein content. $\beta$-Carotene content of $O$. humifusa stem $(195.26 \pm 6.29 \mu \mathrm{g} / 100 \mathrm{~g})$ was about four times higher than that of fruit $(50.83 \pm 0.93 \mu \mathrm{g} / 100 \mathrm{~g}) . \alpha-$ and $\beta$-Tocopherols were found higher in O. humifusa stem while $\gamma$-tocopherol was higher in fruit. Thiamin was not detected in both samples but niacin content in O. humifusa stem was about eight folds $(19.44 \pm 0.03 \mathrm{mg} / 100 \mathrm{~g})$ higher than that of fruit. Folate content in O. humifusa was four times higher in fruit $(23.71 \pm 1.15 \mu \mathrm{g} / 100 \mathrm{~g})$ than stem. Campesterol and $\beta$-sitosterol were found in both stem and fruit. Campesterol contents in stem and fruit were 2.90 and $3.52 \mathrm{mg} / 100 \mathrm{~g}$, respectively; $\beta$-Sitosterol content $(17.50 \pm 1.33 \mathrm{mg} / 100 \mathrm{~g})$ in stem was about two folds higher than that of fruit $(9.19 \pm 0.27 \mathrm{mg} / 100 \mathrm{~g})$. This study shows that both fruits and stem of $O$. humifusa possess beneficial vitamins and phytosterols, hence they can be utilized for the development of nutraceutical products.
\end{abstract}

\section{Vitamin B12 Analysis for Pork Cuts Widely Consumed in South Korea}

\section{Venus Quines* and Jiyeon Chun}

Department of Food Science and Technology, Sunchon National University, South Korea 


\begin{abstract}
Vitamin B12 is known to have important roles in energy metabolism and protein synthesis as a significant component of key enzymes in human body. In South Korea, pork is a major source of vitamin B12, however information on vitamin B12 of the pork cuts favorably consumed in Korea is very limited. Recently, the Korean Rural Development Administration (KRDA) designed a project to investigate vitamin B12 (cyanocobalamin) contents in Korean pork cuts and validate the applied analytical method. Seventeen pork cuts were obtained from first-grade quality hogs based on Livestock Products Act and analyzed for vitamin B12using immune affinity assay coupled with HPLC. Analysis of reference sample BCR487 (pig liver) showed excellent accuracy (100\%) of assay. Results of precision analysis using commercial infant formula showed CV of 5.06\%for repeatability and $8.46 \%$ for reproducibility, which were within the acceptable values of $<15 \%$ and $<32 \%$, respectively, according to AOAC guidelines. The applied method was also subjected to Proficiency Test (PT) by analyzing breakfast cereal which obtained acceptable Z-score. Vitamin B12 contents of pork cuts ranged from 0.42-1.21 $\mu \mathrm{g} / 100 \mathrm{~g}$, with the highest by side bacon and the lowest by rib eye. Rib bacon, the most favorite pork cut, showed vitamin B12 content of $0.80 \mu \mathrm{g} / 100 \mathrm{~g}$. This study provides vitamin B12 database on various pork cuts highly consumed in Korea, which is highly reliable based on method validation and PT results. Retention of vitamin B12 content in individual pork cuts affected by cooking methods would be further needed for vitamin B12 intake.
\end{abstract}

\title{
Simultaneous Quantitative Analytical Method for Sweeteners in Fatty Food
}

\author{
Min Hee Kim ${ }^{1}$, So Na Kim ${ }^{1}$, Su Jeong Lee ${ }^{1}$, Jun Kyu Yang ${ }^{1}$,Ji Yeong Kim ${ }^{1}$,Jang-Hyuk Ahn ${ }^{1,2^{*}}$ \\ ${ }^{1}$ Department of Research E Development, Fore Front TEST, Republic of Korea \\ ${ }^{2}$ Food Science E Technology, CHA University, Republic of Korea
}

\begin{abstract}
Simultaneous quantitative analytical method for 3 kinds of sweeteners in processed food was developed by using rapid sample pre-treatment and HPLC-PDA (High Performance Liquid Chromatography-Photodiode Array Detector). Among various sweeteners, aspartame, saccharin, acesulfame potassium were separated and validated for the analytical method. Since previous simultaneous quantitative analytical method for 3 sweeteners in some fatty processed foods was not possible to perform clean sample preparation, this research was performed for the aim of Korean official method. For the sample pre-treatment, solvent extraction was performed for removing interference effecting fats and centrifugation with weak acid was performed for proteins removal. Ion exchange cartridge was used to capture the sweeteners and to remove residual fats. It was possible to separate the sweeteners using Reversed-phase C18 column ( $5 \mathrm{um}, 4.6 \times 250 \mathrm{~mm}$ ). Calibration curve showed good linearity with high regression coefficients and the result of recovery test showed good recoveries within 90 110\%.
\end{abstract}

\section{Xanthohumol Evaluation in Hop Gun Dry Hopping Techniques}

\author{
Melo A ${ }^{1,4}$, Júlio C. Machado Jr. ${ }^{1}$, Ana Abreu Capitão ${ }^{2}$, Miguel Cancela ${ }^{3}$, Ana Isabel Ribeiro ${ }^{3}$, Catarina Mansilha ${ }^{4}$ and \\ Isabel M.P.L.V.O. Ferreira ${ }^{1}$ \\ ${ }^{1}$ LAQV/REQUIMTE/ Departamento de Ciências Químicas, Laboratório de Bromatologia e Hidrologia, Faculdade de Farmácia - \\ Universidade do Porto, Portugal \\ ${ }^{2}$ Universidade de Aveiro, Campus Universitário de Santiago, Aveiro, Portugal \\ ${ }^{3}$ Unicer-Bebidas SA, Portugal \\ ${ }^{4}$ Departamento de Saúde Ambiental, Instituto Nacional de Saúde Doutor Ricardo Jorge, Rua Alexandre Herculano, Porto, Portugal
}

\begin{abstract}
Dry hopping techniques, also named cold-hopping happens when hops are added at the end of boiling, or even at the cold stages of beer process, fermentation and maturation. This is often used by the breweries to highlight aroma characteristic of hops. It is also known that hops contain a wide polyphenol fraction, include xanthohumol which occurs almost exclusively in hops and are recognized as presenting beneficial health properties, in particular antioxidant, anti-inflammatory, anticancer, immunomodulatory and antimicrobial. In Hop Gun dry hopping techniques the beer is in a maturation tank and remains in flow recirculation with the hops, which is inside an external tank. However, studies about the polyphenol extraction with these techniques are scares. This study evaluated the concentration of xanthohumol with different contact times of beer with hops using Hop Gun dry hopping techniques during maturation.
\end{abstract}

Beer samples were analyzed without recirculation (Control), samples with $12.5 \mathrm{~g}$ of hops per hectoliter with $2 \mathrm{~h}$ (S2) and 
$4 \mathrm{~h}(\mathrm{~S} 4)$ of recirculation, a concentrated sample $(125 \mathrm{~g} / \mathrm{hL})$ with $3.5 \mathrm{~h}$ of recirculation $(\mathrm{CS})$, and a mix with $10 \%$ of CS in the Control (MS).

The Control did not present detectable amounts of xanthohumol, however there was an increase of extraction with more $2 \mathrm{~h}$ of dry hopping recirculation since S2 presented $57( \pm 1) \mu \mathrm{g} / \mathrm{L}$ and S4 $74( \pm 7) \mu \mathrm{g} / \mathrm{L}$ of xanthohumol. The concentrated sample presented a high value of xanthohumol, $419( \pm 22) \mu \mathrm{g} / \mathrm{L}$ and the mix sample presented $53( \pm 1) \mu \mathrm{g} / \mathrm{L}$.

\title{
Impact of Elderberry Addition in Sensory Profile of Bread
}

\author{
Olívia Pinho $^{1,2^{*}}$, Zita E. Martins ${ }^{1}$ and Isabel M.P.L.V.O. Ferreira ${ }^{1}$ \\ ${ }^{1}$ REQUIMTE/ Departamento de Ciências Químicas, Laboratório de Bromatologia e Hidrologia da Faculdade de Farmácia da \\ Universidade do Porto, R. Jorge Viterbo Ferreira 228, Porto, Portugal \\ ${ }^{2}$ Faculdade de Ciências da Nutrição e Alimentação da Universidade do Porto, Rua Dr. Roberto Frias, Porto, Portugal
}

\begin{abstract}
Elderberry and elderberry extracts have been widely studied due to the presence of valuable bioactive compounds, including anthocyanins and fiber. In order to obtain added value bread, elderberry skin, pulp and seeds (EBP) incorporation was tested. EBP was washed, oven-dried, and grinded. Powdered EBP was added to wheat flour at different percentages ( $\mathrm{g} / 100 \mathrm{~g}$ flour): $0 \%$, $5 \%, 7 \%$, and 10\%. Breads were prepared following a bakery recipe and using home-making bread machines (Moulinex, Groupe $\mathrm{SEB}$, France). Bread making program selected included dough preparation ( $2 \mathrm{~h} 20 \mathrm{~min}$ ) and cooking (20 min). The impact of elderberry addition in sensory profile of bread was evaluated based on sensory assays.

A sensory panel composed by 13 members was trained for descriptive analysis according to the guidelines in the ISO 8586 (2012). A list of 20 attributes including global appearance, olfactory and aroma features, texture and overall assessment were selected for breads descriptive profile and a score card was developed to evaluate attribute intensities on $8.5-\mathrm{cm}$ unstructured line scales. Additionally, Triangular test (ISO 4120, 2000) was also applied to the bread samples.

Comparison between sensory profile of control bread (0\% EBP addition) and the other breads added of different amounts of EBP revealed significant differences in all attributes $(\mathrm{p}<0.05)$, except for "Homogeneity" and "Salty". However, regarding "Overall assessment", the mean score was higher for $0 \%$ and $7 \%$ breads. Therefore, EBP can be incorporated at 7\% in bread without compromising the sensory characteristics, contributing to valorization of Elderberry since high antioxidant capacity and higher fiber content is foreseen.
\end{abstract}

\section{Diet Effect on Contaminants Bioaccessibility After in vitro Digestion}

\author{
Miguel Faria*, Armindo Melo and Isabel MPLVO Ferreira
}

LAQV/REQUIMTE/ Departamento de Ciências Químicas, Laboratório de Bromatologia e Hidrologia, Faculdade de Farmácia Universidade do Porto, Portugal

\begin{abstract}
There is nowadays the public awareness that the type of dietary regimen has a strong impact on overall health. Studies supporting the benefits of diets are usually based on its nutritional composition which trended more toward health-beneficial compounds as polyphenolic antioxidants, unsaturated fatty acids, fibers. On the other hand, detrimental constituents in foods (e.g. biological toxins and chemical residues) are usually approached independently from the accompanying constituents of the overall meals. Notwithstanding food components are not ingested individually therefore their effects on the organism should be studied in a context of complex composition. In the present study we have assessed the effects of three widespread dietary regimens (global-average diet; Mediterranean and vegetarian) on the bioaccessibility of the compounds PhiP (2-Amino1-methyl-6-phenylimidazo[4,5-b]pyridine), bezo[a]pirene and ochratoxin A, representatives of the widespread classes of contaminants heterocyclic amines, polycyclic aromatic hydrocarbons and mycotoxins, respectively. Whole meals were spiked with known amounts of the referred compounds and subjected to a standardized enzymatic in vitro digestion including the oral, gastric and intestinal phases. Blank and spiked meals, as well as the bioaccessible fractions of the digests were extracted and concentrated using an acetonitrile-based method and analyzed by HPLC/Fluorescence for the determination of the target compounds. Results indicate that all diets had a strong impact on the overall bioaccessibility of the contaminants and that, for some of the compounds, the type of dietary pattern significantly influences its bioaccessibility. The evaluation of food toxins bioaccessibility after simulated in vitro digestion is thus of major relevance for proper risk assessment.
\end{abstract}




\title{
Optimization of Assisted Ultrasound Osmotic Dehydration of Strawberries Slices in Sucrose Solutions Using Response Surface Methodology
}

\author{
Ali Ferradji, Meriem Bouchareb and Nour el Houda Bouhous \\ High National School of Agronomy, Department of Food Sciences Algiers, Algeria
}

\begin{abstract}
Response surface methodology, according to Central Composite Design (CCD), was used to determine the optimum processing conditions giving maximum water loss and minimum solid gain during osmotic dehydration of strawberries in sucrose solution. The independent variables of osmotic dehydration were temperature $\left(40-65{ }^{\circ} \mathrm{C}\right)$, processing time $(60-180$ $\mathrm{min})$, sugar concentration (45-65\% w/w) and ultrasound (0-45 kHz) Moisture sorption isotherms of the strawberries slices were determined at 25 and $40{ }^{\circ} \mathrm{C}$ by balancing the atmosphere with relative humidity fixed by dilute solutions of sulphuric acid. The optimum conditions were found to be: temperature $=55^{\circ} \mathrm{C}$, time $=180 \mathrm{~min}$, concentration $=60{ }^{\circ}$ Brix and ultrasound at $25 \mathrm{kHz}$. At this optimum point, water loss, weight reduction and solid gain were found to be $84.80 \%$, and $11.16 \%$ respectively. The value of water content of the monomolecular layer $(\mathrm{Xm})$ is $9.95 \%$ at $25{ }^{\circ} \mathrm{C}$ and $6.40 \%$ at $40{ }^{\circ} \mathrm{C}$.
\end{abstract}

\section{Activity of Flavonoids and $\beta$-Carotene During the Auto-Oxidative Deterioration of Food Based O/W Emulsions}

\author{
Sotirios Kiokias* and Theodoros Varzakas \\ Technological Educational Institute of Kalamata, School of Agricultural Technology, Dept. of Food Technology, Greece
}

\begin{abstract}
The replacement of synthetic antioxidants by "safer natural mixtures" is being increasingly advocated nowadays by food industry. This trend has been imposed by the worldwide preference of consumers for the use of natural antioxidants, some of which may exist inherently in foods or be added intentionally during their processing. In this study, the antioxidant effects of certain natural compounds (flavonoids and $\beta$-carotene) were spectrophotometrically assessed during the thermal autooxidation of food relevant oil-in-water emulsions by measuring the formation of primary oxidation products (conjugated dienes and lipid hydroperoxides). Emulsions were preferred over the bulk oil as oxidative systems since they form the basis of many innovative food products and their properties define the quality of the final product to a great extent. At a concentration of $1.5 \mathrm{mmol}$. $\mathrm{kgr}^{-1}$, only quercetin among the tested compounds significantly reduced the oxidative deterioration of cottonseed oil-in-water emulsions. Structural characteristics (positioning of hydroxyl group) or partitioning behavior between the emulsion phases may modulate the flavonoid activity. The high oxygen pressure conditions of the experimental system may explain the lack of any antioxidant activity for $\beta$-carotene. The antioxidant potential of quercetin increased with its concentration until a specific level. On the contrary, the antioxidant concentration within the same tested range $\left(0.75-3 \mathrm{mmol} \mathrm{kgr}^{-1}\right)$ did not impact the activity of catechin $\beta$-carotene. Mixtures of $\beta$-carotene with flavonoids did not exert a tendency for increasing the activity of each individual compound.
\end{abstract}

\section{Preparation and Characterization of Non Heme and Heme Iron Microparticles}

\author{
Carolina Valenzuela ${ }^{{ }^{*}}$, Nadia Rocha ${ }^{1}$ and Fernando Pizarro ${ }^{2}$ \\ ${ }^{1}$ Faculty of Veterinary and Animal Sciences, University of Chile, Santiago, Chile \\ ${ }^{2}$ Micronutrients Laboratory, Institute of Nutrition and Food Technology (INTA), University of Chile, Santiago, Chile
}

\begin{abstract}
The aim of this study was to prepare and characterize microparticles of non heme iron (NHI), and heme iron (HI). Maltodextrin solution was prepared (10\%w/v in deionized water). NHI (iron sulfate) and HI (spray dried blood cells) were suspended individually in maltodextrin solution at 10, 20, 30 and 40\% (w/v), and 5, 10, 15 and 20\% (w/v), and spray-dried; obtaining type A, B, C, D, E, F, G, and H microparticles, respectively. The microparticles were characterized by iron content, efficiency of encapsulation, color, size, and scanning electron microscopy. Eight types of microparticles were obtained. The iron content $(\mathrm{mg} / \mathrm{g})$ of the microparticles was: A $(14.0 \pm 0.3), \mathrm{B}(16.5 \pm 0.7), \mathrm{C}(18.4 \pm 1.1), \mathrm{D}(20.3 \pm 1.1), \mathrm{E}(0.08 \pm 0.02), \mathrm{F}(0.12$ $\pm 0.01), \mathrm{G}(0.15 \pm 0.01)$ and $\mathrm{H}(0.17 \pm 0.01)$. The Fe content increased $(\mathrm{P}<0.05)$ with increasing of NHI or HI concentration.
\end{abstract}


The efficiency of encapsulation (\%) were high: A (78 \pm 6$)$, B (77 \pm 4$), \mathrm{C}(76 \pm 5), \mathrm{D}(78 \pm 3), \mathrm{E}(88 \pm 4), \mathrm{F}(85 \pm 2)$, G (85 \pm 3$)$ and $\mathrm{H}(83 \pm 2)$. HI microparticles showed greater encapsulation efficiency $(\mathrm{P}<0.05)$ than NHI. HI microparticles presented a reddish-brown color and NHI white color. The size $(\mathrm{nm})$ was: A $(918 \pm 17), \mathrm{B}(933 \pm 28), \mathrm{C}(959 \pm 17), \mathrm{D}(981 \pm 7), \mathrm{E}$ $(10,390 \pm 159), F(11,670 \pm 155), G(14,429 \pm 330)$ and $H(16,643 \pm 261)$. The size of the HI microparticles was considerably higher than NHI $(\mathrm{P}<0.05)$. The surface of $\mathrm{HI}$ microparticles was homogeneous, continuous and compact, with spherical shape. NHI microparticles showed a more irregular and heterogeneous surface with depressions and concavities. HI and NHI were effectively encapsulated in maltodextrin microparticles.

\title{
Evaluation of Antioxidant Capacity of Phenolic Compounds of Cactus Purple Pear (Opuntia ficus-Indica) Cultivated in Peru
}

\author{
Coavoy Sánchez Ibeth Anny and Pilco Quesada Silvia* \\ School of Food Engineering, Faculty of Engineering and Architecture, Peruvian Union University, Perú
}

\begin{abstract}
The objective of this research was to evaluate the antioxidant capacity of phenolic compounds of the cactus purple pear (Opuntia ficus-indica) variety most cultivated in Peru specifically in Lima, Huarochirí. To evaluate the total phenol content (mg gallic acid/L sample) and percentage of antioxidant capacity they were considered the following variables: concentration of ethanol $(40 \%$ and $80 \%)$ and temperature $\left(30{ }^{\circ} \mathrm{C}\right.$ and $\left.60{ }^{\circ} \mathrm{C}\right)$. The development of experiments was performed as factorial design 22 with three center points. It is physicochemically characterized the cactus purple pear obtain a $\mathrm{pH}$ of 6.61 , an acidity of 0.06 g. Citric/100 mL sample acid, $11.67{ }^{\circ}$ Brix and maturity index $182.86 \pm 8.96$. Quantification of total phenols was performed according to the Folin-Ciocalteu spectrophotometric method and antioxidant capacity as Mensor et al. (2001). The higher content of phenolics was $1002.47 \mathrm{mg}$ gallic acid/L and a sample of $40.18 \%$ antioxidant at a concentration of $80 \%$ ethanol, and at a temperature of $60^{\circ} \mathrm{C}$.
\end{abstract}

\section{Effect of Atmospheric Cold Plasma on Quality and Shelf Life of Mackerels (Pneumatophorus japonicus)}

\author{
Jing Chen*, Bin Xu, Pin Lin and Shanggui Deng \\ School of Food and Pharmacy, Zhejiang Ocean University, China
}

\begin{abstract}
Atmospheric cold plasma (ACP), an emerging nonthermal technology, possesses numerous advantages over more conventional methods such as low process operational costs, short treatment time at low temperatures, nontoxic nature, significant reduction of water consumption throughout disinfection processes. In this paper, its employment, was evaluated to extend the shelf life of mackerels (Pneumatophorus japonicus), which is highly susceptible to spoilage. Mackerels (Pneumatophorus japonicus) treated with ACP approached the acceptability limit of TVC (6.0 log CFU/g) after $16 \mathrm{~d}$, while samples of the control group exceeded this limit after $8 \mathrm{~d}$. Sensory analyses also confirmed that ACP treatment prolonged the shelf life for about $8 \mathrm{~d}$, compared with samples without treatment. ACP pretreatment also led to lowest TVB-N content and highest springiness and hardness values, indicating that this nonthermal process allowed better quality maintenance. Significant differences $(p<0.05)$ were detected between the PV and TBA values of ACP treated samples and control group, implying obvious inhibitory effect on lipid oxidation. SDS-PAGE analysis and SEM observation also revealed that ACP pretreatment could effectively retard the degradation of myofibrillar proteins and reduce microstructure deterioration. These results demonstrated that ACP is a suitable alternative and presents advantages of increasing the product shelf life.
\end{abstract}

\section{Effect of Super-High Pressure and $\varepsilon$-Poly-L-Lysine Treatment on Skipjack Tuna's Microorganisms and Color during Cold Storage}

\author{
Jian-cong Huo ${ }^{1,3^{*}}$, Shang-gui Deng' and Jian-rong $\mathbf{L i}^{2}$ \\ ${ }^{1}$ Department of Food Science, College of Food Science and Pharmacy, Zhejiang Ocean University, China \\ ${ }^{2}$ College of Chemistry, Chemical Engineering and Food Safety, Bohai University, Jinzhou, China
}


${ }^{3}$ Zhejiang Provincial Key Laboratory of Health Risk Factors for Seafood, China

\begin{abstract}
This study investigated the effect of $\varepsilon$-poly-L-lysine $(\varepsilon-P L)$, with additives, on the microorganisms in skipjack tuna and the effect of super-high pressure on its color after $\varepsilon-P L$ treatment. This is in a bid to optimize storage and lengthen the tuna's shelf life. Results showed that the antimicrobial activity of $\varepsilon-P L$ was positively related to its concentration, and that the optimal concentration appeared to be $0.06 \%$, with kojic acid as an additive comprising $0.1 \%$ in mass and with an immersion time of $20 \mathrm{~min}$. Experiments also revealed that super-high pressure treatment was optimized when the pressure and temperature were kept respectively at $250 \mathrm{MPa}$ and $20^{\circ} \mathrm{C}$ for $30 \mathrm{~min}$. After $20 \mathrm{~min}$ immersion and $30 \mathrm{~min}$ pressure treatment, the sample tended to be stable in brightness, but its yellowness increased steadily while the redness increased a bit before finally decreasing. In general, the experimental samples were better than the control samples in both brightness and redness, and the combined treatment of $\varepsilon-P L$ and super-high pressure significantly decreased microorganisms and constrained discoloration, thus increasing shelf life. Due to the treatment, the tuna's microorganisms did not exceed the limit within $80 \mathrm{hrs}$ and the fish's color remained roughly stable for $40 \mathrm{hrs}$.
\end{abstract}

Citation: : Proceedings of the II International Conference on Food Chemistry \& Technology (FCT-2016).J Food Chem Nanotechnol 2(Suppl 4): S1-S39.

Copyright: This is an Open Access article distributed under the terms of the Creative Commons Attribution 4.0 International License (CC-BY) (http://creativecommons.org/licenses/by/4.0/) which permits commercial use, including reproduction, adaptation, and distribution of the article provided the original author and source are credited. Published by United Scientific Group.

Received: November 28, 2016 Accepted: December 13, 2016 Published: December 15, 2016 